\title{
On-the-fly laser machining: A case study for in-situ balancing of rotative parts
}

\author{
M. Stoesslein \\ PhD Researcher \\ Rolls-Royce Manufacturing and On-Wing University Technology Centre, \\ Faculty of Engineering, University of Nottingham, UK \\ University of Nottingham, \\ Coates Building, Room C31 \\ University Park \\ Nottingham, NG7 2RD \\ moritz@stoesslein.com \\ D. Gilbert \\ EngD Researcher \\ Rolls-Royce Manufacturing and On-Wing University Technology Centre, \\ Faculty of Engineering, University of Nottingham, UK \\ University of Nottingham, \\ Coates Building, Room C31 \\ University Park \\ Nottingham, NG7 2RD \\ epxdg4@exmail.nottingham.ac.uk \\ D. Axinte ${ }^{1}$ \\ Professor \\ Rolls-Royce Manufacturing and On-Wing University Technology Centre, \\ Faculty of Engineering, University of Nottingham, UK \\ University of Nottingham, \\ Coates Building, Room A63 \\ University Park \\ Nottingham, NG7 2RD \\ dragos.axinte@nottingham.ac.uk
}

\footnotetext{
${ }^{1}$ Corresponding Author: Prof. D. Axinte; email: Dragos.Axinte@nottingham.ac.uk
} 
Journal of Manufacturing Science and Engineering

\section{ABSTRACT}

On-the-fly laser machining is defined as a process that aims to generate pockets/patches on target components that are rotated or moved at a constant velocity. Since it is a non-integrated process (i.e. linary/rotary stage system moving the part is independend of that of the laser) it can be deployed to/into large industrial installations to perform in-situ machining, i.e. without the need of disassembly. This allows a high degree of flexibility in it applications (e.g. balancing) and can result in significant cost savings for the user (e.g. no dis(assembly) cost). This paper introduces the concept of on-the-fly laser machining, encompassing, models for generating user defined ablated features as well as error budgeting to understand the sources of errors on this highly dynamic process. Additonally, the paper presents laser pulse placement strategies aimed at increasing the surface finish of the targeted component by reducing the area surface roughness that are possible for on-the-fly laser machining. The overall concept was validated by balancing a rotor system thorough ablation of different pocket shapes by use of a Yb:YAG pulsed fibre laser. In this respect, firstly, two different laser pulse placement strategies (square, hexagonal) were introduced in this research and have been validated on Inconel 718 target material; thus, it was concluded that hexagonal pulse placement reduce surface roughness by up to $17 \%$ compared to the traditional square laser pulse placement. The concept of on-the-fly laser machining has been validated by ablating two different features $(4 \times 60 \mathrm{~mm}$ and $12 \times 4 \mathrm{~mm})$ on a rotative target part at constant speed (100 rpm, $86 \mathrm{rpm})$ in the scope of being balanced. The mass removal of the ablated features to enable online balancing has been achieved within $<4 \mathrm{mg}$. of the predicted value. Additionally, the error modelling revealed that there most of the uncertainties in the dimensions of the feature/pocket orginate from the stability of the rotor speed, which led to the conclusion that for the same mass of material to be removed it is advisable to ablate features (pockets) with longer circumferential dimensions; i.e. stretched and shallower pockets rather than compact and deep. 


\section{INTRODUCTION}

In the last decade the application of advanced materials, such as ceramic composites and superalloys has increased the demand for affordable and capable (micro) material removal techniques to machine difficult-to-cut materials [1]. Thus, the development of a manufacturing process to generate geometric features on such workpiece materials has become a field of great academic and industrial interest especially due to the demands of making precise small components for consumer and industrial products. Without a mechanical contact between the tool and the workpiece, pulsed laser ablation offers many inherent advantages like the absence of tool wear/breakage, chatter, part deflection, and mechanically induced material damage. Nevertheless, laser beam machining (ablation) can also present some disadvantages like the difficulty to control workpiece surface, low material removal rate and microstructure modification of part material; however, these drawbacks could be circumvented by optimising the laser parameters. On this basis, the wide availability of laser ablation systems at a competitive price point led to a quick adaption of this technology across a wide range of industries from the medical to the defence sectors. Consequently, it has established itself as a key enabler to manufacture hard-to-cut materials on a large scale.

On-the-fly laser machining is used to describe a process that is performed on a workpiece that is rotated or moved at a constant velocity during the process. It differs from a laser system/machine on which multiple positioning (linear/rotary) stages are integrated since it only consists of the laser source with its galvanometric beam 
manipulator that then, needs to have its pulse triggering synchronised with the independent motion of the part (belonging to another manufacturing system); such approach would allow the on-the-fly laser machining system to be truly portable and able to perform machining of components after assembly. One very interesting application of the process is on-the-fly depth profiling during ultrashort PLA [2] (i.e. the measuring of the depth of a feature along an arbitrary axis within a single measurement); this approach enables time-gated imaging of samples by evaluating the time-of-flight for light propagating to and from an object during an laser ablation process. Even though not a machining operation per se, this work applies the on-the-fly concept on a laser non-integrated system, which makes it suitable for in-situ interventions (i.e. the component remains in its working environment and is not fully disassembled). Advances in pulse laser ablation (PLA), especially regarding the minimisation of the pulse duration while maximising fluence (laser pulse energy over the area of the spot), have enabled the users to machine features within micron accuracy. However, during the generation of small features, a problem often encountered when using PLA is the phenomena of deep marking, which describes the increased overlap of pulses caused by the mirror accelerating to the defined velocity. Some lasers overcome this issue by using a method called 'sky-writing', where the laser beam scanning speed is accelerated to a constant target velocity before the marking process starts [3]; this however, reduces the operational time and limits the range of motion of the laser beam. Neverthless, on-the-fly laser machining, offers the ability to ablate a rotating component by synchronizing the rotor with the laser system. Jaeggi [3] 
developed such a method for $2.5 \mathrm{D}$ processing of rotatives, a practice often used for surface and structuring applications. It achieved a pulse positioning precision of $1 \mu \mathrm{m}$ with the target component rotating at 510 RPM; however, the research lacks a detailed analysis on the errors in different features and how this would affect the use of such system in other applications like in-situ balancing. A similar but more basic approach to the problem of in-situ balancing was lead by J. F. Walton et al. [4]. The method with the aim to balance rotatives using pulse laser ablation is described, however, the paper fails to to show any actual results nor does it give any guide on the achievable accuracy and precision of the method; so, it could hardly be a base for further advancements in the field. Additionally, the implemented controls only account for point ablation, a process at which the laser pulses at the exact location of the imbalance effectively drilling a hole to remove material, which can damage the structural integrity of complex geometry components and lead to reduced life time especially when balancing larger components where the removal of several grams of material is necessary.

The errors associated with a machining process allow the user to predict whether the process will be able to provide the required accuracy for generating a predefined geometric feature. Thus, an analysis of errors is essential to all manufacturing processes to validate their suitability for a selected design. Previous studies have focused on the error budgeting multi-axis lasers [5] and polar coordinate laser writing systems [6]. The polar coordinate laser writing system uses a rotary encoder to coordinate the motorised air-bearing spindle with the purpose of achieving writing accuracy in the order of $0.1 \mu \mathrm{m}$ at rotational speed of up to $800 \mathrm{rpm}$. This work 
analyses the system, from an error perspective, on a pulse basis rather than from the point of view of feature generation to investigate the overall effect of errors on fabricating diffractive optical elements. Further research has been done on the accuracy of 3D laser scanning systems [7], which focused on defining the random and systematic error in the laser scanner head, as well as, developing an empirical model to account for the systematic error. Another laser application relevant to the present paper that has received a detailed error analysis is the terrestrial laser scanning (TLS) [8]. The paper proposes a new method to analyse errors, referred to as angular error analysis, which offers a more complete analysis of the positional accuracy. It is important to realise that analysing the error in the placement of the pulses does not give a complete picture of the resultant 3D geometry if applied to a feature. One also has to consider what effects the errors have on the material removal rate and, therefore, on the feature as a whole. For example, errors may affect the total amount of material removed by a laser machining process, if pulses are placed on zones of the component outside the focal length of the laser. This is also true if the beam incidence changes or the targeted component's surface is not even, which can have an effect on ablation characteristic as shown by D. S. George [9]. Hence, for a more complete approach to on-the-fly laser machining, the errors of the machined pockets/features and their impact on the process neeed to be considered. Thus, the error budgeting of on-the-fly laser machining has yet not been studied to the extent necessary to make informed decisions, potentially hindering its growth in industry and research. 
Nowadays many design problems can be solved using an iterative process and are therefore, prime candidates for computer optimisation algorithms. Laser machining especially, enables the operator to define a large number of parameters (beam power, pulse duration, frequency etc.) and hence, the optimum results depend on the experience of the user or the time available to trial several combinations. Thus, academic focus has been on using algorithms to determine the optimum set of parameters for a variety of applications. K. Erkorkmaz et al. [10] utilised the optimisation techniques to define the laser pulse trajectory, which minimises the process time. This enabled them to obtain the ideal path by considering tool and process constraints. However, it required the position of all pulses to be known for the shape to be generated. Another interesting example of exploiting the power of computer based optimisation algorithms in regards to lasers is the search for critical parameters for a laser welding process [11]. This study establishes relationships between depth of laser penetration, bead width (i.e. width of the weld filler material), tensile strength of the welded component and the beam power, velocity and focal position of the laser. It then uses a genetic algorithm (GA) in order to establish the optimum solutions. Similarly, another paper [12] utilizes the newly developed 'teachinglearning-based optimization algorithm' in order to establish optimum solutions for ultrasonic machining, abrasive jet machining and wire electrical discharge machining. The same has been done for laser fusion processes [13], where the author designs a 'fast laser cutting optimization algorithm' based on limiting the design parameters in order to limit the solution space. Another laser cutting optimisation study [14] for 
Nd:YAG lasers cutting thin superalloy sheet focused its attention on minimising the kerf width, taper and deviation using a hybrid approach of the Taguchi methodology and the grey relational analysis. The author considered the following parameters: oxygen pressure, pulse width, pulse frequency and cutting speed. An important observation was in reference to the difference between the optimum parameters for a straight cut and a curved cut, which had to be determined separately. Besides, a model of Nd:YAG microgrooving of Al2TiO5 based on artificial neural network methodology [15] has been developed, which found a good agreement between the parameters determined by the algorithm and of the validated work. Hence, while some laser machining processes have been extensively studied using optimisation algorithms in order to obtain the ideal parameters, on-the-fly machining using PLA has not yet been fully studied, particularly the errors in generating a desired 3D feature.

\subsection{Scope of the paper}

This paper presents a mathematical model that predicts the material removal, process time and the errors of on-the-fly laser machining, of a specified geometrical area targeting as application the balancing of a rotating part at constant velocity. The model needs an initial calibration to find the response of the workpiece material to a fixed set of energetic parameters and then using the kinematics of the laser beam and of the part, it ensures that a specified geometry/volume of the workpiece (rotating part) is removed by laser ablation at a high degree of robustness. The validation of the model is presented using a special rig with a part to be balanced made of Inconel 718, which makes the demonstration of relevance for applications in aerospace industry. The model 
also finds its use in supporting a decision to remove a given volume of material (to enable the balancing to a desired grade) in such a way that it minimises the errors; this is done by associating to the model an error budget that quantifies the error in the critical dimensions of the volume to be removed of the proposed on-the-fly balancing system.

\section{THE CONCEPT OF ON-THE-FLY LASER MACHINING}

During the recent decade, ample research has been done on pulse laser ablation (PLA) and its thermal and kinematic effects on the surfaces of components [16]. Machining on-the-fly using PLA does not have an a priori full integration between the galvanometric manipulator of the laser beam and the moving/rotating stages of the part to be machined; this requires not only engineering integration of the two systems but also deep understanding of sources of errors that might impede on the time synchronisation of the beam action and the moving part. As on-the-fly pulse laser machining is defined as ablating the targeted component while it is moving or rotating at a constant velocity using a non-integrated laser system, it finds its use in repair tasks of industrial installations without the need of their disassembly, i.e. in-situ repair. However, in this approach the accuracy of the individual pulse placement affects the overall accuracy of an ablated feature; therefore, its accuracy is dependent on the errors in the individual spot placement on the target rotating/moving part. Figure 1 presents a schematic of the principle that governs the on-the-fly laser balancing concept on which the galvanometric actuated mirror can manipulate the beam in the $\mathrm{x}$ and $\mathrm{y}$ directions; the rotating movement $(\omega)$ of the part is to be integrated with the laser 
triggering and the beam galvanometer. For simplicity reasons, in the following, the onthe-fly concept is applied that the beam will be manipulated (at intervals) only in the $x$ direction, while the rotation $(\omega)$ of the rotor will be used to imitate the raster path of the beam to generate the ablated feature (as presented in the insert Figure 1); hence, the error related to the intermittent spot placement in the $\mathrm{x}$ direction will be considered small enough to be neglected in the proposed models. However, the errors related to spot placement along y $\left(D_{y}\right)$, circumferential direction is the combination of errors of the following quantities (see Figure 1): the frequency of pulses ( $\Delta$ f); the time to trigger a laser pulse $\left(\Delta \mathrm{t}_{d}\right)$; the rotor radius $(\Delta R)$; the spot diameter $(\Delta \phi)$; the velocity of the moving component $(\Delta \omega)$. Thus, by superimposing single spots on y (circumferential) and $x$ direction laser ablated pockets/features can be generated on the moving/rotating components. This is to be repeated several times, where each complete cycle is regarded as a layer; henceforth, the total number of layers $\left(n_{z}\right)$ of the feature affect its depth $(d)$. In this context, it is of crucial importance to study the errors related to these interdependencies upon the geometrical accuracy of the ablated feature and allow the necessary actions to enable high precision removal of material on the target rotating part.

Once, the model for on-the-fly laser machining/balancing is understood and the errors budgeted, it allows the comparison of different shapes (e.g. is it more accurate to ablate a long patch along the curvature of the rotor or a wide patch) and their geometrical accuracies and thus, select the geometry of the ablated feature that has a minimal error from the targeted one. 
While the concept of on-the-fly laser machining is exemplified for a rotating disc at a constant velocity, it can also be applied to any scenario of part moving arbitrarily within the scanning area of the laser beam.

\section{MODELLING THE ERRORS FOR ON-THE-FLY LASER MACHINING TARGETING AN APPLICATION FOR IN-SITU BALANCING}

The model determines the optimum pulse frequency $(f)$, laser power $(P)$ and number of ablated layers $\left(n_{z}\right)$ for a defined geometric feature using multi-objective optimisation techniques in order to minimise the process time $(t)$ and the deviation from the desired mass removal $(m)$. Furthermore, the cumulative error of critical dimension of the feature (i.e. $D_{y}$ - see Figure 1 ) resulting from pulse placement error is calculated for the optimised parameters.

Figure 2 shows a flowchart of the on-the-fly laser machining strategy introduced in this work, which starts with the selection of the critical dimensions $\left(D_{x}, D_{y}\right)$ of the required feature as well as the desired mass $(m)$ to be removed by on the-fly machining; note that the depth $(d)$ of the feature (see Figure 1 ) is not defined but results from the chosen removal mass (which depends on the specified balancing grade). The desired feature is optimised for the minimum machining time whilst meeting the mass removal criteria using an optimisation algorithm (see Section 3.1). To model the mass removal $(m)$ and determine the process time $(t)$, a grid for the pulse placement is generated (see Section 3.2), then a single footprint is simulated and applied to the grid (see Section 3.3). The grid geometry is adjusted according to the output of the scaling model relying on an artificial intelligence technique accounting for errors occurring at 
beam/workpiece interface, e.g. variation of material redeposition (see Section 3.4). Lastly, the on-the-fly machining errors of the feature associated with the optimum parameters (i.e. the output of the optimisation study) are determined (see Section 3.5).

\subsection{Optimization modelling minimizing the processing time while meeting the mass} removal requirement

The multi-objective genetic optimisation algorithm is a search method, which attempts to find the optimum parameters of the on-the-fly laser machining for which both goals, i.e. process time and error of the mass removal, are minimised, however they cannot reach an absolute minima at the same time; those solutions can be obtained via genetic optimisation (represented as a Pareto curve), which has been considered the approach in this work. Compared to other optimisation algorithms used in engineering problems, genetic optimisation has few requirements and allows for a global search (i.e. it attempts to find the global minimum rather than the local minimum) [11], [12], [17].

The genetic optimisation algorithm employed in this work follows the wellestablished steps in genetic optimisation algorithms [18] as outlined below:

1. (i) Formulate an equation for each goal (i.e. the processing time and error in removal mass) describing the problem with the variable parameters (i.e. the fitness function). (ii) Chose a size of the population of the on-the-fly laser machining parameters and other variables of the multi-objective genetic optimisation algorithm (i.e. crossover and mutation functions, crossover probability). (iii) Initialise a random population of the pre-determined size. (iv) 
Set a time limit and maximum allowed number of generations before the algorithm finishes (i.e. the finishing criteria).

2. Evaluate each member of the population of the on-the-fly process parameters and choose the best members (also known as parents) as the elite to be carried on to the next generation. A generation is defined as the all members of one population. Therefore, at each iteration of the algorithm, one new generation is created on basis of the previous one.

3. Check whether finishing criteria of the on-the-fly laser machining algorithm is reached (e.g. limits on computational time, generations).

4. Reproduce the current population members and repeat the algorithm until one finishing criteria is fulfilled. The reproduction is done by either mutating one parent or crossing the parameters of two parents.

As the steps 1-4 are common in the field of process optimisation, they are not detailed here but used only to support the proposed on-the-fly strategy.

With this in mind, a multi-objective genetic optimisation algorithm [19] is used to optimise the process defining parameters (pulse frequency - $f$, laser power - $P$, number of layers $-n_{z}$ and the pulse spacing in $\mathrm{x}$ and $\mathrm{y}-d_{x, y}$ ) for on-the-fly laser machining to meet the two algorithm goals: (i) minimum process time ( $t$ ); (ii) minimum error of the mass removal $(m)$. Furthermore, some practical constraints had to be imposed for the genetic optimisation: 
- The pulse frequency $(f)$ is limited by the constraints of the scaling model (see Section 3.4, Table 1 ) and the laser system being used, where $f_{\min }$ is the minimum and $f_{\max }$ the maximum pulse frequency.

$$
f_{\min } \leq f \leq f_{\max }
$$

- The laser power $(P)$ is limited by the material ablation threshold, $P_{\min }$, and the maximum value $P_{\max }$ to avoid metallurgical damage of the part.

$$
P_{\min } \leq P \leq P_{\max }
$$

- The number of layers $\left(n_{z}\right)$ is limited to a minimum of 1 and a maximum, $n_{z_{-} m a x}$ chosen based on the desired removal mass.

$$
1 \leq n_{z} \leq n_{z_{-} \max }
$$

- The pulse spacing $\left(d_{x, y}\right)$ is limited by the minimum $\left(\omega_{\min }\right)$ and maximum $\left(\omega_{\max }\right)$ rotational velocity permissible by the motor and its controller, and the pulse frequency $(f)$. Additionally, the constraints imposed by the calibration process of the scaling model limit the pulse spacing (see Section 3.4, Table 1).

$$
\frac{2 \pi R\left(\frac{\omega_{\min }}{60}\right)}{f_{\max }} \leq d_{y, x} \leq \frac{2 \pi R\left(\frac{\omega_{\max }}{60}\right)}{f_{\min }}
$$

- The pulse energy (i.e. the laser power - P, divided by the frequency - $f$ ) is limited by the calibration process of the scaling model, which enables the determination of the ablation depth of a laser process (see Section 3.4, Table 1). 


$$
\frac{P}{f}=E
$$

Note that the pulse frequency $f(\mathrm{~Hz})$, pulse spacing $d_{y, x}(\mu \mathrm{m})$ and the number of passes $n_{z}$ have been constrained to integers. The laser power $(P)$ has its value expressed to 1 decimal place.

\subsection{Generation of the grid coordinates}

To generate a feature, a number of laser pulses need to be placed in a manner to form the required shape; the coordinates of all necessary laser pulses are regarded to as a grid within this work. All grids consist of a number of tracks (i.e. lines of pulses) orientated along the circular curvature of the targeted part, i.e. y-axis (see Figure 1). Thus, each track corresponds to a single laser ablation trigger, i.e. one revolution of the rotating part. The spacing between the individual pulses $\left(d_{x, y}\right)$ determines the amount of pulses $\left(n_{y}\right)$ per line as well as the number of tracks $\left(n_{x}\right)$ as shown in Eq. (6); where $D_{x, y}$ is the critical dimension and $\phi$ the diameter of an individual laser pulse. The critical dimension is defined as the measurement that describes the feature in the relevant direction (see Figure 3). For example, for a rectangular shape it is the length in the $y$ direction $\left(D_{y}\right)$ and the width in the $x$ direction $\left(D_{x}\right)$.

$$
n_{x, y}=\frac{D_{x, y}-2 \phi}{d_{x, y}}+1
$$

In this work it is assumed that the targeted component rotates with a constant velocity $(\omega)$. Therefore, the time between the triggering and the start of the ablation process $\left(t_{d}\right)$ is expressed as the circumferential distance on the rotor as shown in Eq. (7), 
where $\omega$ the rotational velocity of the targeted component and $R$ the radius of the rotor.

$$
d_{y t}=t_{d}\left(\frac{\omega}{60}\right)(2 \pi) R
$$

The vertical pulse spacing $\left(d_{y}\right)$ between the individual pulses is governed by the rotational velocity of targeted component $(\omega)$ the frequency of the laser pulses $(f)$ and the radius of rotor $(R)$ as shown in Eq. (8). The horizontal pulse spacing $\left(d_{x}\right)$ is solely limited by the accuracy achievable of the laser positioning system (galvanometer). During the generation of each track, the mirror of the laser head/galvanometer remains stationary in the $\mathrm{x}$ and $\mathrm{y}$ axes.

$$
d_{y}=\frac{\left(\frac{\omega}{60}\right)(2 \pi)}{f} R
$$

Hence, the length $D_{y}$ of the feature can be expressed as shown in Eq. (9).

$$
D_{y}=\sum_{i=2}^{n_{y}} \frac{\left(\frac{\omega}{60}\right)(2 \pi)}{f} R+\phi
$$

Some on-the-fly laser machining applications (e.g. balancing) could be employed to high value components, where it is essential to ensure that minimal damage (e.g. metallurgical transformation) is caused by the pulsed laser material removal process, which can also include various forms of micro and macro scale morophological changes, like increased surface roughness [20]. Therefore, to minimises surface roughness one has to ensure that the fluence is equally distributed over the ablation area; this can is achieved by placing the pulses using the hexagonal packaging technique, which has been conclusively shown to be the densest distribution of laser footprints [21] (i.e. 
offering the most equal distribution of fluence) compared to the traditionally used square packaging of pulses (see Figure 3).

\subsection{Generating a laser footprint}

There are previous works regarding surface prediction through energy beam material removal that have adopted a crater-by-crater approach for modelling the evolution of the surface texture [22]-[24]. A common theme in these approaches is to first generate a single crater from process parameters, and then to overlap/convolute this, either with/without crater modification for subsequent pulses, to achieve a complete machined surface. This paper proposes to utilise this method in predicting the radius of a single crater for any given input parameters; this is building on the previous experience in the group by Gilbert et al [22], where calibration between the laser system and target material is carried out prior to use. The plotted relationship between energetic parameters of the laser ablation process and their corresponding material removal when used to ablate a known target material is of logarithmic nature. This relationship is then used to calculate further depths of ablation at other energetic parameters.

The energetic parameters used are expressed as a normalized fluence $\left(\Theta_{\text {normalized }}\right)$. This is simply a ratio between the fluence calculated from the energetic parameters in question - $\Theta$ (see Equation (10)), and the maximum ones achievable by the laser system - $\Theta_{0}$ (see Equation (11) and (12)).

$$
\Theta=\frac{8 P}{\pi f\left(2 r_{z}\right)^{2}}
$$




$$
\begin{gathered}
\Theta_{0}=\frac{8 P_{\max }}{\pi f_{0}\left(2 r_{0}\right)^{2}} \\
\Theta_{\text {normalised }}=\frac{\Theta}{\Theta_{0}}
\end{gathered}
$$

Where additional notations have the following meaning: $P_{\max }-$ maximum average power output of the laser $(\mathrm{W}) ; f_{o}$ - lowest pulse repetition frequency before attenuation; $r_{z}$ - beam radius at workpiece surface with a focal length of $z$.

Figure 4 shows a generic relationship between ablated depth and normalized fluence following Equation (13). The vertical dotted line shows the normalized threshold fluence $\Theta_{\text {th }}$ at which ablation begins to occur using the specified laser system and target material. Thus, it is possible using this information to calculate the radial position within the laser beam at which this threshold fluence $\Theta_{t h}$ value is reached and therefore, the target material ablated.

$$
d_{s}=a \ln \left(\frac{\Theta}{\Theta_{0}}\right)+b
$$

Using the equation (13), the threshold fluence (where $d=0$, see Equation (14) and (15)) can be calculated;

$$
\begin{gathered}
0=a \cdot \ln \left(\frac{\Theta}{\Theta_{0}}\right)+b \\
e^{\left(-\frac{b}{a}\right)}=\frac{\Theta}{\Theta_{0}}
\end{gathered}
$$

Once this normalised fluence value is known, by using a known beam energy distribution, it can be used to derive the radial position from the beam centre at which threshold fluence occurs (see Figure 5). This radial position across the laser footprint will therefore, indicate the region outside which the ablated depth could be considered 
zero. This is shown graphically in Figure 5 where an example of a generic laser beam energy profile is coaxially aligned with its corresponding ablated crater (excluding the zone where the fluence is below the threshold $\left.-\Theta_{t h}\right)$.

Using the above described crater of the laser this can be placed on the grid of pulses as described in Section 3.2 to enable the prediction of the ablated feature.

\subsection{Development of an intelligent scaling model to predict the material removal rate}

\section{of pulse laser ablation}

With ablation of metals it is likely that some degree of material redeposition, i.e. melt expelled from the crater and solidified around the footprint, occurs [14], [22], [25]. As this phenomenon is random and in repeated ablated layers is likely to affect the prediction accuracy of the model, this aspect has been addressed by a scaling approach, i.e. compensating the accuracy of the ablation depth with the number of layers to minimise the effect of the material redeposition. As the scope of this paper is about controlling the on-the-fly laser machining this approach was considered appropriate to enable the proof of the proposed in-situ balancing concept. Thus, for the scaling approach for the laser footprint (see Section 3.3) an artificial neural network (ANN), see Figure 6, was utilised. This research uses the network to predict the real (affected by material redeposition) ablation depth $(d)$, which is dependent on the process parameters (pulse frequency $-f$, pulse spacing $-d_{x, y}$, the critical dimensions of the feature - $D_{x}$ and $D_{y}$, and number of layers $\left.-n_{z}\right)$. These parameters are the input neurons into the ANN, while the neurons within the hidden layers act as processing units containing the transfer function (Eq. (16), where $x$ is the sum of all randomly weighted 
inputs of the neuron) to obtain the output, i.e. ablation depth (d); note that Eq. 16 is mathematically equivalent to a hyperbolic tangent function, however runs faster by sacrificing some accuracy to computational speed and is therefore, commonly used in neural network designs [26]. All other factors, which may influence ablation depth like material, pulse energy, distance to focal plane and spot size are kept constant within this work.

$$
y=\frac{2}{1+e^{-2 x}}-1
$$

The network was designed using the Levenberg-Marquardt backpropagation algorithm [27] which minimizes the computing time by approximating the Hessian matrix and calculating the Jacobian matrix using backpropagation; since it is a commonly used algorithm in neural network design, it is only briefly introduced.

\subsection{Error modelling using a standard deviation approach}

With the models ready to predict the optimum removal rate for a given feature to address on-the-fly laser machining, and bearing in mind that this can be utilised for in-situ balancing of rotatives, an obvious question is the influence of the variances of the process parameters upon the obtained ablated geometry.

Considering that the width (Dx) is commanded by the laser galvanometer sequentially between ablated tracks, this section presents a way to determine the expected errors in the length (Dy) of the ablated pocket along the cord of the rotor; thus, with the ablation depth known from ANN (see section 3.4), Dx considered of negligible variation, the variance of the on-the-fly ablated volume can be obtained enabling the assessment of the precision of the in-situ laser balancing method. This 
allows the development of a general error prediction model, which is not tied to one specific experimental set-up. In this respect, each pulse is affected by up to three errors: (i) error in the circumferential direction (i.e. y error); (ii) error in the horizontal direction (i.e. $x$ error); (iii) error in depth (i.e. d error).

The errors are calculated using the multi-variable calculus approach [28] as shown in Equation (17), where $\Delta_{H}$ is describing the total error and $A, B$ and $C$ are variables, contributing to $H$, for which the error values are known. This is known to deliver the most accurate prediction of the actual error. Furthermore, it allows one to study the individual impact of the each contributing error parameter $(A, B, C \ldots)$ and therefore, therefore to identify ways reduce the variability of the output (Z).

$$
\Delta_{H}=\sqrt{\left(\frac{\partial Z}{\partial A}\right)^{2} \cdot\left(\Delta_{A}\right)^{2}+\left(\frac{\partial Z}{\partial B}\right)^{2} \cdot\left(\Delta_{B}\right)^{2}+\left(\frac{\partial Z}{\partial C}\right)^{2} \cdot\left(\Delta_{C}\right)^{2}+\cdots}
$$

Therefore, the total error in y $\left(\Delta D_{y}\right)$ is expressed by Eq. (18), which describes the combined error due to the time lag $\left(\Delta t_{d}\right)$, which determines the starting position of each track, and the sums of all pulse placement errors within one track (based on Eq. (7), (8) and (9)).

$$
\Delta D_{y}=\sqrt{\left(\Delta d_{y t}\right)^{2}+\Delta\left(\left(\sum_{i=2}^{n_{y}} d_{y}\right)+\phi\right)^{2}}
$$

Hence, the overall error is dependent on the error in the time lag $\left(\Delta t_{d}\right)$, the pulse frequency $(\Delta f)$, the pulse radius $(\Delta \phi)$, the rotor radius $(\Delta R)$ and the rotational velocity $(\Delta \omega)$ (see Figure 7). 
In this work, the $x$ error is only influenced by the accuracy of the chosen galvanometric laser head $\left(\Delta D_{x}\right)$ while the $z$ (or $\mathrm{d}$ ) error is solely dependent on the accuracy of the ANN in predicting the depth $(d)$. Hence, it is obtained by assuming a Gaussian distribution of the ablated depth predictions by the ANN. Thus, the error in mass $(\Delta m)$, which is obtained using Eq. (19) and (20), is calculated.

$$
\begin{gathered}
m=D_{y} \cdot D_{x} \cdot d \cdot \rho \\
\Delta m=\sqrt{\frac{\partial m^{2}}{\partial D_{y}} \cdot\left(\Delta D_{y}\right)^{2}+\frac{\partial m^{2}}{\partial D_{x}} \cdot\left(\Delta D_{x}\right)^{2}+\frac{\partial m^{2}}{\partial d} \cdot(\Delta d)^{2}}
\end{gathered}
$$

In conclusion, by knowing the errors affecting on-the-fly laser machining, the user could have a strict control over the desired feature generation in terms of dimensions and mass removed. This is necessary for processes with stringent design requirements or applications, which require an accuracte mass removal like balancing.

\subsection{On-the-fly laser machining workflow}

Considering the previous modelling specifications, the procedure to perform onthe-fly laser machining relies on the following steps:

1. The user specifies the desired feature with critical dimension $\left(D_{x}\right.$ and $\left.D_{y}\right)$ and mass removal target $(m)$; note that critical dimensions $\left(D_{x}\right.$ and $\left.D_{y}\right)$ depend on the space available on the part, between existing geometrical features of the rotative, to enable the removal of the required mass $(m)$ leading to the desired level of balancing. 
2. The optimisation algorithm generates an initial population of process parameters (pulse frequency $-f$, pulse spacing $-d_{x, y}$ and number of passes $-n_{z}$ ) using the supplied constraints (see Equation (1) - (5)).

3. Each member of the population is evaluated:

- $\quad$ Pulse coordinates for the specified feature are generated (see Equation (6) - (9)).

- The laser footprint is simulated for the chosen energetic parameters (see Equation (10) - (15)).

- The simulated laser footprint is plotted for each of the coordinates generated in step 3.1.

- The simulated feature is scaled based on the model outputs from the ANN (see Section 3.4).

4. The optimization algorithm checks for exit conditions (i.e. maximum number of generations, computational time limit). If none of the conditions is met, continue with step 2.

5. The associated errors of the feature are evaluated (see Equation (18)) to enable corrective actions to the on-the-fly laser machining process.

\section{METHODOLOGY AND EXPERIMENTAL DESIGN}

For all trials a Yb:YAG SPI G3.1 SM fibre laser with a maximum average output power $(P)$ of $20 \mathrm{~W}$ and a pulse duration ranging from 9-220 ns with a wavelength of $1062 \pm 2 \mathrm{~nm}$ was used. It has an operating range between 1 and $500 \mathrm{kHz}(f)$ and the beam quality, $M^{2}$, has been specified as 1.8 by the manufacturer. The actual maximum average power output $(P)$ was measured to be approx. $17.24 \mathrm{~W}$ when demanding the 
full $20 \mathrm{~W}$ from the system (mean value over 100 samples). Lowering the frequency $(f)$ below $35 \mathrm{kHz}$ limits the pulse energy to prevent damage to the equipment (i.e. maximum pulse energy $(E)$ at maximum power is achieved at $35 \mathrm{kHz}(f)$ and $20 \mathrm{~W}(P)$ for a 220 ns pulse duration). The spot diameter $(\phi)$ was measured to be approximately 72 $\mu \mathrm{m}$ on the focal plane using a complementary metal-oxide semiconductor (CMOS) beam profiler camera.

\subsection{Calibration trials}

\subsubsection{Optimization model}

Before validation and utilization for on-the-fly laser machining, the previously developed model (see Section 3) had to be calibrated. This will provide the process parameters to achieve the desired mass removal target at a minimum process time. For this, the optimisation algorithm (see Section 3.1) needs to be configured. Thus, a population size of 100 for process parameters $\left(f, P, n_{z}\right.$ and $\left.d_{x, y}\right)$ and two goals $(t, m)$ was chosen. The elite count was set to two (i.e. the number of members who are guaranteed to survive into the next generation), while the crossover probability is 0.8 . The maximum number of generations (i.e. the limit) was chosen to be 300 ; this ensured a justifiable computing expense. New mutations were created using Gaussian distribution in accordance with the standard genetic optimisation practice [29].

\subsubsection{Laser spot footprint model for on-the-fly laser machining}

The footprint model (see Section 3.3) needed to be calibrated in order to obtain the radius of the laser spot at which the ablation threshold occurs for a given material (i.e. Inconel 718). This was done by varying the fluence levels $(\Theta)$ and measuring the depth 
$\left(d_{s}\right)$ of the footprint from which the logarithmic dependence as in Eq. (21) was obtained (considering the $\Theta_{0}=2021 \mathrm{~J} / \mathrm{cm}^{2}$ ).

$$
d_{s}=1.0206 \cdot \ln \left(\Theta_{\text {normalised }}\right)+1.467
$$

Hence, using the laser parameters (laser power - $P$, pulse frequency $-f$, beam radius $-r_{z}$, and focal position $-z$ ) and assuming a Gaussian beam profile, the footprint of one laser pulse can be determined. Then, the diameter of the spot $(\phi)$ can be measured (see Figure 5).

\subsubsection{Scaling model}

Having obtained the spot radius and plotted the pulses onto the generated grid (see Fig $\mathrm{x}$ ), the ANN needs to be calibrated to obtain the predicted feature depth $\left(d_{p}\right)$. The optimum neural network structure (see Section 3.4) for this example consists of one hidden layer containing six neurons (see Figure 6). The input layer has five neurons (i.e. frequency $-f$, pulse spacing $-d_{x, y}$, number of layers $-n_{z}$, critical dimensions $-D_{x}$ and $D_{y}$ ) and the output layer has one neuron (i.e. depth of the feature - $d$ ). It has been found that increasing the number of neurons above the determined optimum of six causes over-fitting due to the noisy input data due to phenomena like shielding, which are difficult to control within the experimental setup of this work.

The network was trained using 57 trials by varying in the pulse frequency $(f)$, pulse spacing $\left(d_{x, y}\right)$, critical dimensions $\left(D_{x}\right.$ and $\left.D_{y}\right)$ and number of layers $\left(n_{z}\right)$ (see Table 2 Experimental and training results); in order to simplify this model, the pulse spacing $\left(d_{y, x}\right)$ has been kept equal in both $\mathrm{x}$ and $\mathrm{y}$ directions. All laser ablated samples have been 
carried out on Inconel 718 as workpiece material at a constant pulse energy of $400 \mathrm{~mJ}$ (see Eq. (22)).

$$
E=\frac{P}{f}=400 \mathrm{~mJ}
$$

The scan speed, $v_{\text {scan, }}$ was determined by Eq. (23).

$$
v_{\text {scan }}=d_{x, y} f
$$

The sample depth $\left(d_{m}\right)$ was evaluated using a white light interferometer (Bruker

\section{Contour GT).}

In order to validate the designed network, the data samples were randomly divided into three categories:

- Training samples that were used to train the network and continuously adjust the error between the measured and predicted depth.

- Validation samples that were used to measure the generalization and stop the algorithm if there are no more significant improvements.

- Testing samples that were used to validate independently the performance of the developed network. They have no effect on the training of the network.

For this work, the data laser ablated samples were split into: $70 \%$ for training, $15 \%$ for validation and $15 \%$ for testing.

The limitations of the ANN (see Table 1) are defined by the range of samples submitted to the training of the network, which were selected by considering the restrictions imposed by the laser system and material (i.e. Inconel 718) as well as the expected application of the network. 
The designed network achieved a reasonable accuracy (see Figure 8) in predicting the depth $\left(d_{p}\right)$ of an ablated rectangle on Inconel 718 as long as the limitations of the network were taken into consideration (see Table 1). Figure 8 presents the training, validation and testing samples all at a mean squared error below $11 \mu \mathrm{m}$.

Due to the highly noisy calibration data (when measuring the depth - $d$ - using a white light interferometer) this could be considered a well trained network.

The ANN was trained with the goal of a zero mean squared error. An error (i.e. an indicator of the performance of the network) of 1.0929 at 10 epochs was achieved (see Figure 8).

\subsection{Experimental trials for the validation of pulse placement strategy}

The first stage of the validation trials was aimed to quantify the effect of the square and hexagonal pulse placement grid strategy (see Fig. 3) that will further support the on-the-fly laser machining concept. Laser ablation creates features by overlapping several pulses in a geometrical pattern forming the desired feature (e.g. rectangle) with the chosen critical dimensions ( $D_{x}$ and $D_{y}$ in this example). By adapting the pulse placement strategy to result in smooth pockets (i.e. a low surface roughness) so that post machining processes can be reduced or eliminated. This concept was validated on static target surfaces $(4 \times 7 \mathrm{~mm}$ ) made of Inconel 718 by comparing square and hexagonal pulse placement strategies when using the following process parameters: a total of 350 pulses per track $\left(n_{y}\right)$ over a total of 200 tracks $\left(n_{x}\right)$, i.e. spacing between pulses and tracks of $20 \mu \mathrm{m}\left(d_{x, y}\right)$, with 1 and 3 layers $\left(n_{z}\right)$ have been ablated using the 
Yd:YAG laser with a frequency of $35 \mathrm{kHz}(f)$, a power of $17 \mathrm{~W}(P)$, pulse duration of 220 ns and a scanspeed of $525 \mathrm{~mm} / \mathrm{s}\left(v_{\text {scan }}\right)$.

To measure only the surface roughness caused by the laser machining process in the two pulse placement grid strategies, a white light interferometer (Bruker Contour GT) was used to determine root mean square area roughness measurement (Sq) specified in ISO 25178 [30].

Using hexagonal pulse placement compared to the traditional square placement (see Section 3.2, Figure 3) during PLA showed to minimise the surface area roughness. After one layer, a reduction in the root mean square area roughness $(\mathrm{Sq})$ of $17 \%$ was achieved when utilising hexagonal pulse placement strategy; after three layers there was still an improvement of $13 \%$ (see Table 3 ).

Figure 9 shows that the hexagonal pulse placement strategy leads to a smoother surface. This is attributed to the more evenly distributed fluence due to the hexagonal placement of pulses. This is supported by Figure 9, which shows for square placement (in Figure $9 \mathrm{c}$ and d) visible tracks while hexagonal placement results in a significantly less visible ablation tracks (in Figure $9 \mathrm{a}$ and b). Especially, in time critical processes (e.g. on-the-fly balancing during maintenance of high value components), where each laser ablation layer can take up to several minutes machining time, the ability to affect the surface finish easily can save time and potentially an additional finishing or polishing machining process.

\subsection{Experimental trials for on-the-fly laser machining}


To conduct the material removal and error prediction trials, a test rig consisting of a shaft with a $Q 140 \mathrm{~mm}$ disc mounted by ball bearing on an experimental stand (see Figure 10) has been considered as the part to be laser on-the-fly machined. The rotor is driven using a 42 W DC Maxon motor (max. speed $1200 \mathrm{rpm}$ ), which is kept at a constant velocity using an external PID motor controller (Maxon Epos 24/2), and connected to the shaft using a flexible coupling.

Two different on-the-fly laser machining processes were completed in order to validate the material removal and error prediction models for a user specified feature, i.e. a rectangle with chosen critical dimensions $D_{x}$ and $D_{y}$ (see Table 4). Henceforth, the dimensions of the two rectangles were chosen in a manner to have one long (i.e. a large $D_{y}$, Ablated feature A) rectangle along the curvature of the rotor and one wide (i.e. $D_{x}>$ $D_{y}$, Ablated feature B) rectangle.

As an ablation target, a small Inconel 718 sheet $(80 \times 20 \times 0.6 \mathrm{~mm})$ has been firmly attached to the rotor of the testing rig. The sample's weight $(m)$ has been determined using a scale with an accuracy of $2 \mathrm{mg}$ in order to verify, after ablation, the accuracy of the material removal prediction after the on-the-fly laser machining process. For this, the on-the-fly strategy introduced in Section 3 was used to determine the suitable process parameters (pulse frequency $-f$, laser power $-P$, pulse spacing $-d_{x, y}$, number of layers $-n_{z}$, number of tracks $-n_{x}$, pulses per track - $n_{y}$ and rotational velocity $\omega)$. The minimum permissible rotational velocity of the rotor $(\omega)$ was set at $60 \mathrm{rpm}$, since for speeds below $60 \mathrm{rpm}$ the velocity control became unstable (i.e. high variations, due to the high inertia of the rotor). As an optimisation target for the chosen feature, an 
arbitrary desired mass removal target $\left(m_{d}\right)$ with a permissible mass variation $\left(\Delta m_{d}\right)$ has been assigned to each ablated feature. Therefore, the optimisation algorithm choses process parameters, which results in the desired feature with the chosen mass removal $(m)$ within an accuracy of $\Delta m_{d}$ and a minimum process time $(t)$.

All errors necessary for the on-the-fly laser machining error budgeting model were obtained using empirical means or from the provided documentation of the manufacturer. The error in the angular velocity $(\Delta \omega)$, the time delay $\left(\Delta t_{d}\right)$, the feature depth $(\Delta d)$ using the ANN, the rotor radius $(\Delta R)$, as well as, the pulse diameter $(\Delta \phi)$ were obtained using empirical means as shown in Table 5.

The error in the frequency $(\Delta f)$ and in the $\mathrm{x}$ mirror for the beam positioning $\left(\Delta D_{x}\right)$ were obtained using the manufacture's data sheets. The error in the rotor speed $(\Delta \omega)$ has shown to be of variable mangnitude depending on the speed of the motor (see Figure 11).

Hence, the speed has been measured over a time period of approx. 100 seconds at $60,200,400,600,900$ and $1200 \mathrm{rpm}$. The error has then been calculated using three standar deviations and a $5^{\text {th }}$ degree polynomial has been fitted (see Figure Figure 11 and Equation (24)).

$$
\Delta \omega= \pm\left(-4.2 \times 10^{-14} \omega^{5}+1.6 \times 10^{-10} \omega^{4}-2.3 \times 10^{-7} \omega^{3}+0.00017 \omega^{2}-0.065 \omega+15\right) \cdot 100
$$

\section{MODEL VALIDATION AND DISCUSSION}

The model was validated with a focus on potential applications in the in-situ balancing of components using pulse laser ablation by targeting to remove material 
covering rectangular pockets (i.e. features). In this section, the material removal and error prediction concept to manufacture features for on-the-fly laser machining using Inconel 718 are studied using two examples.

\subsection{Evaluation of the proposed material removal model}

The on-the-fly laser machining feature prediction model was run using the chosen parameters for the two sets of scan trials (see Section 4 and Table 4). The two ablated features (see Figure 12) differ in the width and length (i.e. the critical dimensions $D_{x}$ and $D_{y}$ ) as well as the desired mass removal - $m$ (see Table 4). This further enables the comparison between different orientated features and how it affects the effectivity of on-the-fly laser machining (i.e. process time and material removal rate). The optimised parameters for the predicted process duration $(t)$ and mass removal $\left(m_{p}\right)$ are shown in Table 6. Afterwards, both ablated features using the hexagonal pulse placement strategy, have been validated on the testing rig with the attached Inconel 718 sheet (see Section 4.3 and Figure 12).

The process time of ablated feature $A(4 \times 60 \mathrm{~mm})$ and ablated feature $B(12 \times 4$ $\mathrm{mm}$ ) vary widely; this is due to the number of triggers necessary to achieve the specified feature (i.e. number of tracks $-n_{x}$ multiplied by number of passes $-n_{z}$ ).

Hence, particularly long features in the circumferential direction benefit from on-the-fly machining, while shorter features however, can decrease the material removal rate per time unit significantly. Hence, for example for balancing a longer (circumferential length, e.g. ablated feature A) feature will significantly decrease process time $(t)$ for a desired mass removal $(m)$ target. 
Due to errors mentioned in section 3.5, the $60 \times 4 \mathrm{~mm}$ area (i.e. ablated feature A) removed $85 \mathrm{mg}$ of material, with a target removal of $83.69 \mathrm{mg}$ (see Table 7). This is an underestimate of $1.31 \mathrm{mg}(-1.38 \%)$. The $4 \times 12 \mathrm{~mm}$ area (i.e. ablated feature $\mathrm{B}$ ) removed $13 \mathrm{mg}$ of material with a target removal of $16.25 \mathrm{mg}$, an overestimate of 3.25 mg (+20.00\%). The ablated feature B showed much larger errors due to the higher influence of on-the-fly errors on shapes with wider $D_{x}$ than $D_{y}$ (see Section 5.3). This is due to the higher number of revolutions (i.e. laser triggers) needed to create the feature (i.e. number of tracks $-n_{x}$ multiplied by number of passes $-n_{z}$ ), where each possess another possibility of a laser misfire. Additionally, variations in the weight measurement (e.g. dust on the sample or scale, human error, etc.) have a bigger impact on smaller masses if measured as a percentage. The specific scale used for these trials had an error of approx. $\pm 2 \mathrm{mg}$.

To conclude, on basis of the higher errors for wide ablated features (high $D_{x}$, e.g. ablated feature B), on-the-fly laser machining is more effective when used for long ablated features (high $D_{y}$, e.g. ablated feature A) due to the lower number of laser triggers and therefore, decreased error potential and process time and higher material removal rate. In a balancing scenario, ablated feature $A$ would have been chosen as it outperforms ablated feature B in all measured aspects (material removal rate, process time and error).

\subsection{Evaluation of the error budgeting model}

Utilising on-the-fly laser machining for balancing components requires the user to have a good understanding of how to optimise the laser processing parameters and 
characteristics of the ablated feature in order to maximise the material removal and minimise process time (see Section 5.2). However, it also calls for an understanding of the associated errors of on-the-fly laser machining and therefore the accuracy of the feature predictions as well as the achievable tolerances using this process. Henceforth, the developed error prediction model (see Section 3.5 and Equation (18) and (20)) for on-the-fly laser machining was validated on ablated feature A and B.

As shown in Table 8 the errors are all within the predicted range. The measured length $(D y)$ and mass $(m)$ are stated at a 95\% confidence level (coverage factor $k=2$ ). Therefore, the error prediction model demonstrates its capabilities to predict accurately errors for the length $\left(D_{y p}\right)$ and the mass $\left(m_{p}\right)$ of the feature. of on-the-fly laser machining processes. Below is a demonstration of its analytical capabilities on basis of the example introduced in section 5.2.

Figure 13 shows the individual error contributions to the overall circumferential length error $\left(\Delta D_{y}\right)$ in this specific example. Ablated feature A's error is mainly contributed by the velocity of the rotor $(\Delta \omega)$ with over $95.6 \%$ in total. There is a similar trend for ablated feature B with $95.8 \%$ contribution by the rotor velocity $(\Delta \omega)$. The error of the rotor radius $(\Delta R)$ contributes another $4.4 \%$ and $3.97 \%$ to ablated feature $A$ and $\mathrm{B}$ respectively. Spot size $(\Delta \phi)$, time delay $\left(\Delta t_{d}\right)$ and pulse frequency $(\Delta f)$ are negligible. Therefore, improvements in the accuracy of the circumferential length $\left(\Delta D_{y}\right)$ can be achieved by increasing the stability of the motor control. As shown in Figure 11, increasing the rotor speed $(\omega)$ can also lead to better circumferential accuracy (due to a lower velocity error). However, this may cause an increase of the pulse spacing $\left(d_{x, y}\right)$ or 
decrease of the pulse energy $(E)$ (see Eq. (5) and (8)). Therefore, if the material removal rate is to be kept, this requires a different laser system capable of constant pulse energies $(E)$ at higher pulse frequencies $(f)$. Also, increasing the ablated track length $\left(D_{y}\right)$ increases the rotor manufacturing accuracy $(\Delta R)$ impact.

Hence, it is summarized that the error increases with circumferential length $\left(D_{y}\right)$ of the ablated area. This is due to the high contribution of the rotor velocity $(\Delta \omega)$ in this example. Increasing the stability of the motor control at low speeds ( $<100 \mathrm{rpm}$ ) will make the manufacturing quality of the rig (i.e. rotor radius $(\Delta R)$ ) become more prevalent when considering errors from the on-the-fly machining process.

The error in mass of material removed $-\Delta m$ (see Table 8) showed a good agreement between the predicted and measured mass removal error $(\Delta m)$. Figure 14 shows the overall error contribution to the mass error $(\Delta m)$. Ablated feature A and B have a similar high error contribution from the depth error $(\Delta d)$ with $95.5 \%$ and $94.7 \%$ respectively. The error in the critical dimension $D_{x}$ is negligible for both ablated features. However, the error in the second critical dimension $D_{y}$ shows to be more prevalent if the surface area of the ablated feature is smaller ( $4.5 \%$ for ablated feature $A$ with a surface area of $240 \mathrm{~mm}^{2}$ and $5.3 \%$ for ablated feature B with a surface area of $48 \mathrm{~mm}^{2}$ ). However, if the error in the rotor speed $(\Delta \omega)$ is neglected the error contribution of $D_{y}$ to the mass error $(\Delta m)$ becomes negligible. Overall, the error for ablated feature $A$ is $13.1 \%$ while it is $13.2 \%$ for ablated feature B. Hence, for in-situ balancing processes ablated feature A allows for a higher mass removal $(m)$, shorter process time $(t)$ while the error in the mass removal $(\Delta m)$ does not vary greatly. 
To conclude, the error model enables the prediction of inaccuracies in the critical dimensions and mass removal of the feature generated using on-the-fly laser machining. The highest contributor is the error in the feature depth $(\Delta d)$. However, if taking the said errors into consideration, the on-the-fly laser machining model enables valuable predictions for in-situ balancing applications. Hence, in a balancing scenario, where it is important to weigh accuracy of the feature shape used for mass removal and overall process time, a feature similar to ablated feature A would offer the best compromise between a high mass removal rate and a minimum error to achieve such.

\section{CONCLUSIONS}

The rise of pulse laser ablation (PLA) machining in industry and academia led to several new manufacturing techniques, one of them being on-the-fly machining using PLA. Its main advantages are the ability to machine rotatives with precision, accuracy, speed and little need for skilled labour. However, while many laser machining processes have received a great amount academic attention, on-the-fly pulsed laser machining so far has gone unnoticed. Specifically, its application potential as a correction method during balancing has so far been overlooked, also due to the non-existence of a reliable error budgeting model on a feature basis to predict the inaccuracies of a feature and hence evaluate on-the-fly laser machining as a potential manufacturing process. This papers presents a model, which is capable of predicting material removal (exemplified on Inconel 718 test pieces) and processing time of a specified feature generated by onthe-fly laser machining. 
- A model has been developed for predicting the material removal and process time for on-the-fly laser machining. The model combines analytical approaches with artificial intelligence to approximate the material removal. It has shown to offer a high accuracy with a maximum error below $3 \mathrm{mg}$ when prediciting optimum process parameters to generate features of Inconel 718 using on-thefly laser machining.

- A model to predict errors in the feature occurring from the on-the-fly laser machining process has been developed and validated using two sets of on-the-fly laser machining trials where it succeeded in accurately predicting the inaccuracies. Furthermore, the model allowed insights into the origin of the errors and henceforth, the stability of the motor control has been identified as the main source for errors (approx. 95\% contribution) to the error of the critical dimension $D_{y}$. For the overall mass, the error in the depth of the feature $(\Delta d)$ has the highest contribution (approx. 95\%).

- It has been shown that by changing the pulse placement strategy from the traditional square placement to the denser hexagonal placement, allows the fluence to be more evenly distributed over the ablation area and therefore reduces the surface roughness of the on-the-fly laser machining process. Improvements between $12 \%$ and $17 \%$ could be observed.

- The model has been successfully applied and validated on a testing rig. Two trials have been conducted, one focusing on a long circumferential ablation feature, while to other one focused on a wide but short ablation feature. The mass 
differences observed were $-1 \%$ and $+20 \%$ respectively. It has been concluded that the higher error in the wide trial stems from the high number of tracks, which increases the errors of on-the-fly laser machining.

To conclude, the models enables operators to accurately machine features, assuming a well calibrated laser machining system on an industrial scale. Additionally, the methodology can be used as a corrective method for balancing rotatives in-situ due to the low space requirements of a fibre laser as well as the instantaneous vaporisation of waste material compared to "traditional" balancing methods.

\section{ACKNOWEDGMENTS}

The authors would like to acknowledge the generous support of Rolls-Royce plc, and especially to Mr. Peter Winton and Mr. Mike Harrison as well as the EPSRC for the CASE award scholarship. Furthermore, we would like to thank Stewart Lowth for his guidance and help while designing the testing rig.

\section{FUNDING}

This research was funded by an CASE award scholarship of the EPSRC. 


\section{NOMENCLATURE}

\begin{tabular}{|c|c|}
\hline$P$ & Power of the laser \\
\hline$\phi$ & Spot diameter $\left(1 / \mathrm{e}^{2}\right)$ \\
\hline$r_{z}$ & Beam radius \\
\hline$R$ & Radius of the rotor \\
\hline$m$ & Removed material \\
\hline$m_{p}$ & Predicted material removal \\
\hline$m_{d}$ & Desired material removal \\
\hline$d$ & Depth of the laser ablated pocket \\
\hline$d_{m}$ & Measured depth of the laser ablated pocket \\
\hline$d_{p}$ & Predicted depth of the laser ablated pocket \\
\hline$d_{s}$ & Depth of the laser spot \\
\hline$\omega$ & Angular velocity of the rotating part \\
\hline$f$ & Frequency of the laser \\
\hline$n_{x}$ & Number of laser tracks \\
\hline$n_{y}$ & Number of laser pulses per track \\
\hline$n_{z}$ & Number of laser passes \\
\hline$t_{d}$ & Time delay between triggering and firing of the laser \\
\hline$E$ & Laser pulse energy \\
\hline$\Theta$ & Laser output fluence \\
\hline
\end{tabular}




\section{REFERENCES}

[1] A. K. Dubey and V. Yadava, "Laser beam machining-A review," Int. J. Mach. Tools Manuf., vol. 48, no. 6, pp. 609-628, May 2008.

[2] R. Lausten and P. Balling, "On-the-fly depth profiling during ablation with ultrashort laser pulses: A tool for accurate micromachining and laser surgery," Appl. Phys. Lett., vol. 79, no. 6, p. 884, Aug. 2001.

[3] B. Jaeggi, B. Neuenschwander, T. Meier, M. Zimmermann, and G. Hennig, "High Precision Surface Structuring with Ultra-Short Laser Pulses and Synchronized Mechanical Axes," Phys. Procedia, vol. 41, pp. 319-326, Jan. 2013.

[4] J. F. Walton, M. Cronin, and R. Mehta, "Advanced balancing using laser machining," SAE, Aerosp. Technol. Conf. Expo., vol. -1, Sep. 1991.

[5] X. Cheng, Y. Huang, S. Zhou, J. Liu, and X. Yang, "Study on the generative design method and error budget of a novel desktop multi-axis laser machine for micro tool fabrications," Int. J. Adv. Manuf. Technol., vol. 60, no. 5-8, pp. 545-552, Sep. 2011.

[6] A. G. Poleshchuk, V. P. Korolkov, V. V. Cherkashin, S. Reichelt, and J. H. Burge, "Polar-coordinate laser writing systems: error analysis of fabricated DOEs," in International Symposium on Optical Science and Technology, 2001, pp. 161-172.

[7] H.-Y. Feng, Y. Liu, and F. Xi, "Analysis of digitizing errors of a laser scanning system," Precis. Eng., vol. 25, no. 3, pp. 185-191, Jul. 2001.

[8] A. Cuartero, J. Armesto, P. G. Rodríguez, and P. Arias, "Error analysis of terrestrial laser scanning data by means of spherical statistics and 3D graphs.," Sensors (Basel)., vol. 10, no. 11, pp. 10128-45, Jan. 2010.

[9] D. S. George, A. Onischenko, and A. S. Holmes, "On the angular dependence of focused laser ablation by nanosecond pulses in solgel and polymer materials," Appl. Phys. Lett., vol. 84, no. 10, p. 1680, Mar. 2004.

[10] K. Erkorkmaz, A. Alzaydi, A. Elfizy, and S. Engin, "Time-optimized hole sequence planning for 5-axis on-the-fly laser drilling," CIRP Ann. - Manuf. Technol., Apr. 2014.

[11] P. Sathiya, K. Panneerselvam, and M. Y. Abdul Jaleel, "Optimization of laser welding process parameters for super austenitic stainless steel using artificial neural networks and genetic algorithm," Mater. Des., vol. 36, pp. 490-498, Apr. 2012.

[12] R. Venkata Rao and V. D. Kalyankar, "Parameter optimization of modern machining processes using teaching-learning-based optimization algorithm," Eng. Appl. Artif. Intell., vol. 26, no. 1, pp. 524-531, Jan. 2013.

[13] B. Adelmann and R. Hellmann, "Fast Laser Cutting Optimization Algorithm," Phys. 
Procedia, vol. 12, pp. 591-598, 2011.

[14] R. Rao and V. Yadava, "Multi-objective optimization of Nd:YAG laser cutting of thin superalloy sheet using grey relational analysis with entropy measurement," Opt. Laser Technol., vol. 41, no. 8, pp. 922-930, Nov. 2009.

[15] D. Dhupal, B. Doloi, and B. Bhattacharyya, "Optimization of process parameters of $\mathrm{Nd}$ :YAG laser microgrooving of Al2TiO5 ceramic material by response surface methodology and artificial neural network algorithm," Proc. Inst. Mech. Eng. Part B J. Eng. Manuf., vol. 221, no. 8, pp. 1341-1350, Jan. 2007.

[16] A. K. Dubey and V. Yadava, "Experimental study of Nd:YAG laser beam machining-An overview," J. Mater. Process. Technol., vol. 195, no. 1-3, pp. 1526, Jan. 2008.

[17] O. B. Nakhjavani and M. Ghoreishi, "Multi Criteria Optimization of Laser Percussion Drilling Process Using Artificial Neural Network Model Combined with Genetic Algorithm," Feb. 2007.

[18] M. Mitchell, An Introduction to Genetic Algorithms. London, England: A Bradford Book The MIT Press, 1996.

[19] K. Deb, Multi-Objective Optimization Using Evolutionary Algorithms. John Wiley \& Sons, 2001.

[20] A. Kar and J. Mazumder, "Two-dimensional model for material damage due to melting and vaporization during laser irradiation," J. Appl. Phys., vol. 68, no. 8, p. 3884, 1990.

[21] H.-C. Chang and L.-C. Wang, "A Simple Proof of Thue's Theorem on Circle Packing," 2010. [Online]. Available: http://arxiv.org/pdf/1009.4322v1.pdf. [Accessed: 03-Jun-2015].

[22] D. Gilbert, M. Stoesslein, D. Axinte, P. Butler-Smith, and J. Kell, "A time based method for predicting the workpiece surface micro-topography under pulsed laser ablation," J. Mater. Process. Technol., vol. 214, no. 12, pp. 3077-3088, Dec. 2014.

[23] M. C. Kong, C. B. Miron, D. A. Axinte, S. Davies, and J. Kell, "On the relationship between the dynamics of the power density and workpiece surface texture in pulsed laser ablation," CIRP Ann. - Manuf. Technol., vol. 61, no. 1, pp. 203-206, Jan. 2012.

[24] J. Billingham, C. B. Miron, D. A. Axinte, and M. C. Kong, "Mathematical modelling of abrasive waterjet footprints for arbitrarily moving jets: Part II-Overlapped single and multiple straight paths," Int. J. Mach. Tools Manuf., vol. 68, pp. 30-39, May 2013.

[25] A. Sharma and V. Yadava, "Modelling and optimization of cut quality during pulsed Nd:YAG laser cutting of thin Al-alloy sheet for straight profile," Opt. Laser 
Technol., vol. 44, no. 1, pp. 159-168, Feb. 2012.

[26] M. Dorofki, A. Elshafie, and O. Jaafar, "Comparison of artificial neural network transfer functions abilities to simulate extreme runoff data," ... Chem. Biol. ..., 2012.

[27] H. Yu and B. Wilamowski, "Levenberg-marquardt training," Ind. Electron. Handb., 2011.

[28] I. G. Hughes and T. P. A. Hase, "Measurements and their uncertainties," 2010.

[29] MathWorks, "How the Genetic Algorithm Works," Matlab Documentation, 2015. [Online]. Available: http://uk.mathworks.com/help/gads/how-the-geneticalgorithm-works.html. [Accessed: 15-Nov-2015].

[30] International Organization for Standardization, "ISO 25178-2:2012 Geometrical product specifications (GPS) -- Surface texture: Areal -- Part 2: Terms, definitions and surface texture parameters," 2012. 


\section{Figure Captions List}

Fig. 1 Schematic of on-the-fly pulse laser ablation with main sources of errors

Fig. 2 Flowchart of the on-the-fly laser machining approach

Fig. 3 Example of pulse placement grids for a rectangle using hexagonal and square pulse placement.

Fig. 4 Example calibrated relationship between depth of ablation and normalised fluence

Fig. 5 Example of laser beam energy distribution (top) and crater depth profile (bottom), with the red shaded area indicating fluence below the ablation threshold level

Fig. 6 Schematic of the artificial neural network for the prediction of the ablation depth in on-the-fly laser machining

Fig. 7 Schematic of the error sources in circumferential (y) direction

Fig. $8 \quad$ Validation performance of the trained neural network showing the point on which minimum error between the predicted and measured ablated depth was achieved

Fig. 9 Comparison between the surface finishes of a) 1 layer hexagonal pulse placement, b) 3 layers hexagonal pulse placement, c) 1 layer square pulse placement, d) 3 layers square pulse placement

Fig. $10 \quad$ View of the testing rig setup 
Journal of Manufacturing Science and Engineering

Fig. $11 \quad$ Velocity error dependent on rotor speed

Fig. 12 Rotary stage with Inconel 718 sample attached (see experimental set-up in Figure 10)

Fig. 13 Error contribution of ablated feature A and B to $\Delta$ Dy (see Eq. (18))

Fig. 14 The error contribution of ablated feature $A$ and $B$ to $\Delta m$ (see Eq. (20)) 


\section{Table Caption List}

Table $1 \quad$ Variation intervals for the neural network input parameters

Table 2 Experimental and training results of the neural network for Inconel 718 calibration trials, blue shading indicates square ablated patches (i.e. $4 \times 4$ $\mathrm{mm}$ ), while orange shading indicates rectangular (i.e. not square) ablated patches

Table 3 Root mean square (Sq) area roughness (ISO 25178) comparison between hexagonal and square pulse placement

Table $4 \quad$ Parameters for the on-the-fly laser machining and error prediction trials

Table $5 \quad$ Errors associated with the pulse generation and placement

Table 6 On-the-fly laser machining process parameter optimisation results for ablated feature $A$ and $B$

Table 7 On-the-fly laser machining results for ablated feature $A$ and $B$

Table 8 Error model evaluation results for $\Delta \mathrm{D}_{\mathrm{y}}$ and $\Delta \mathrm{m}$ 


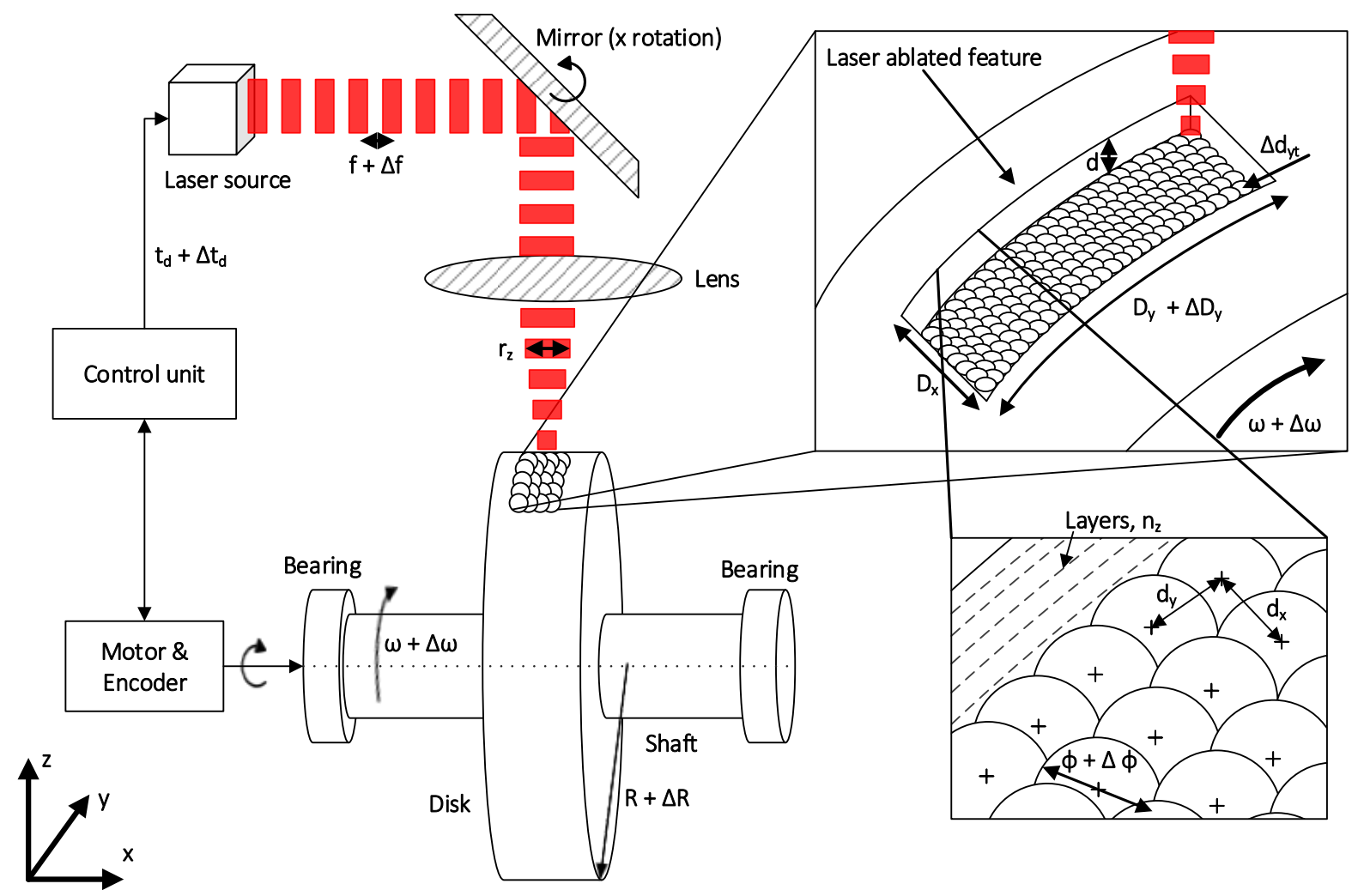

Figure 1 Schematic of on-the-fly pulse laser ablation with main sources of errors 
Journal of Manufacturing Science and Engineering

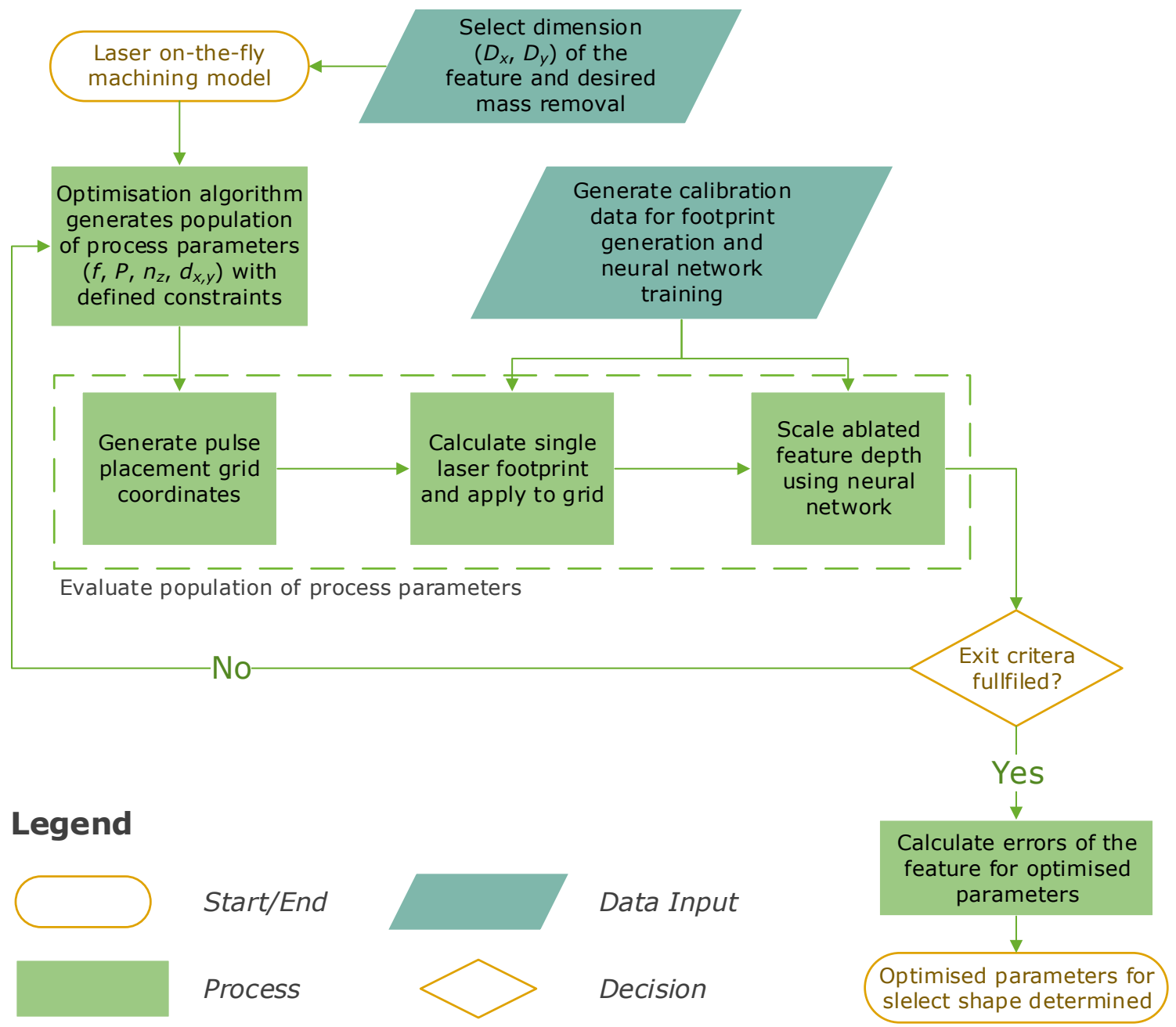

Figure 2 Flowchart of the on-the-fly laser machining approach 
Journal of Manufacturing Science and Engineering

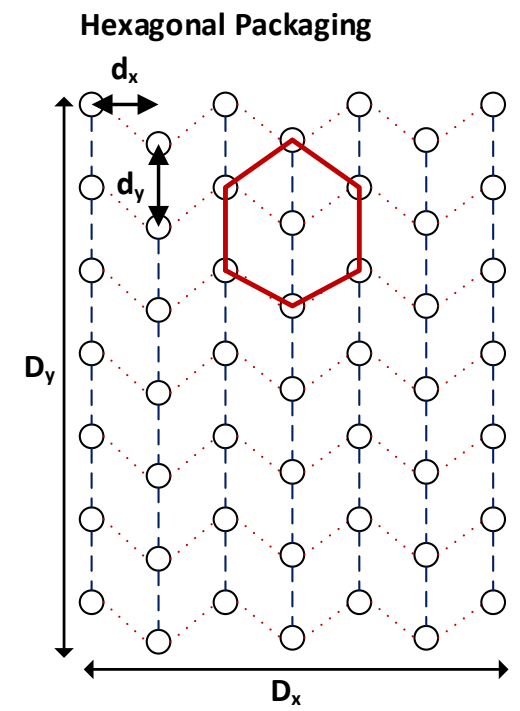

\section{Square Packaging (Traditional)}

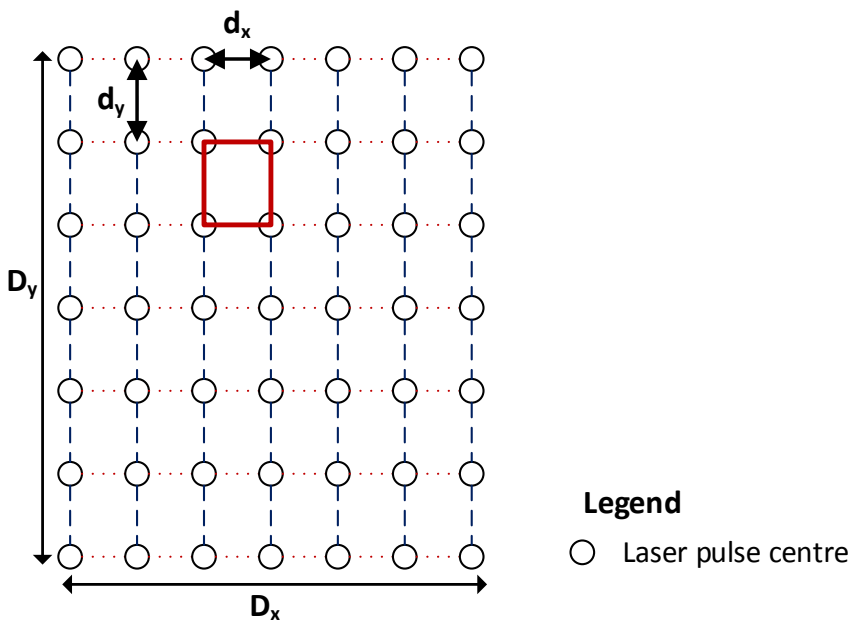

Figure 3 Example of pulse placement grids for a rectangle using hexagonal and square pulse placement 
Journal of Manufacturing Science and Engineering

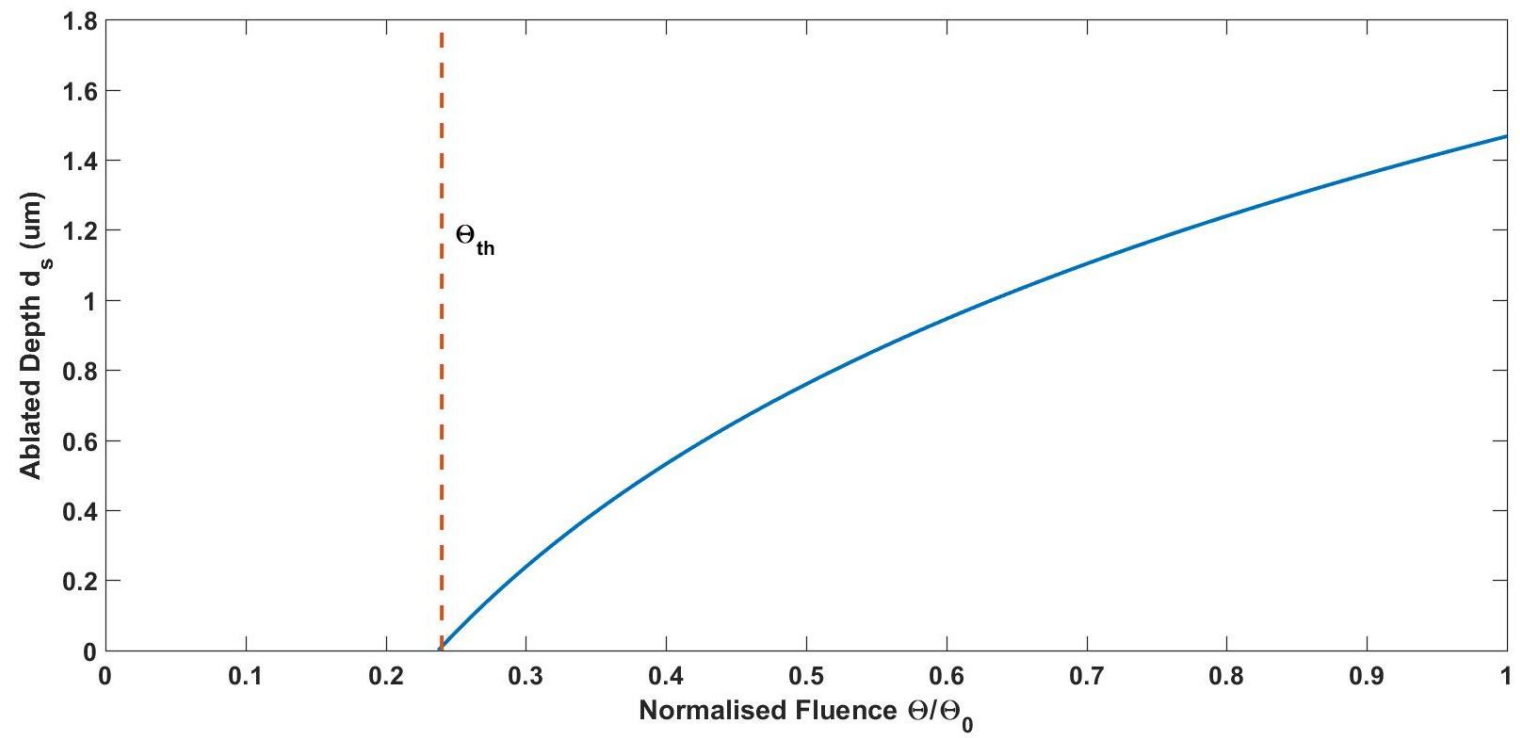

Figure 4 Example calibrated relationship between depth of ablation and normalised fluence 
Journal of Manufacturing Science and Engineering

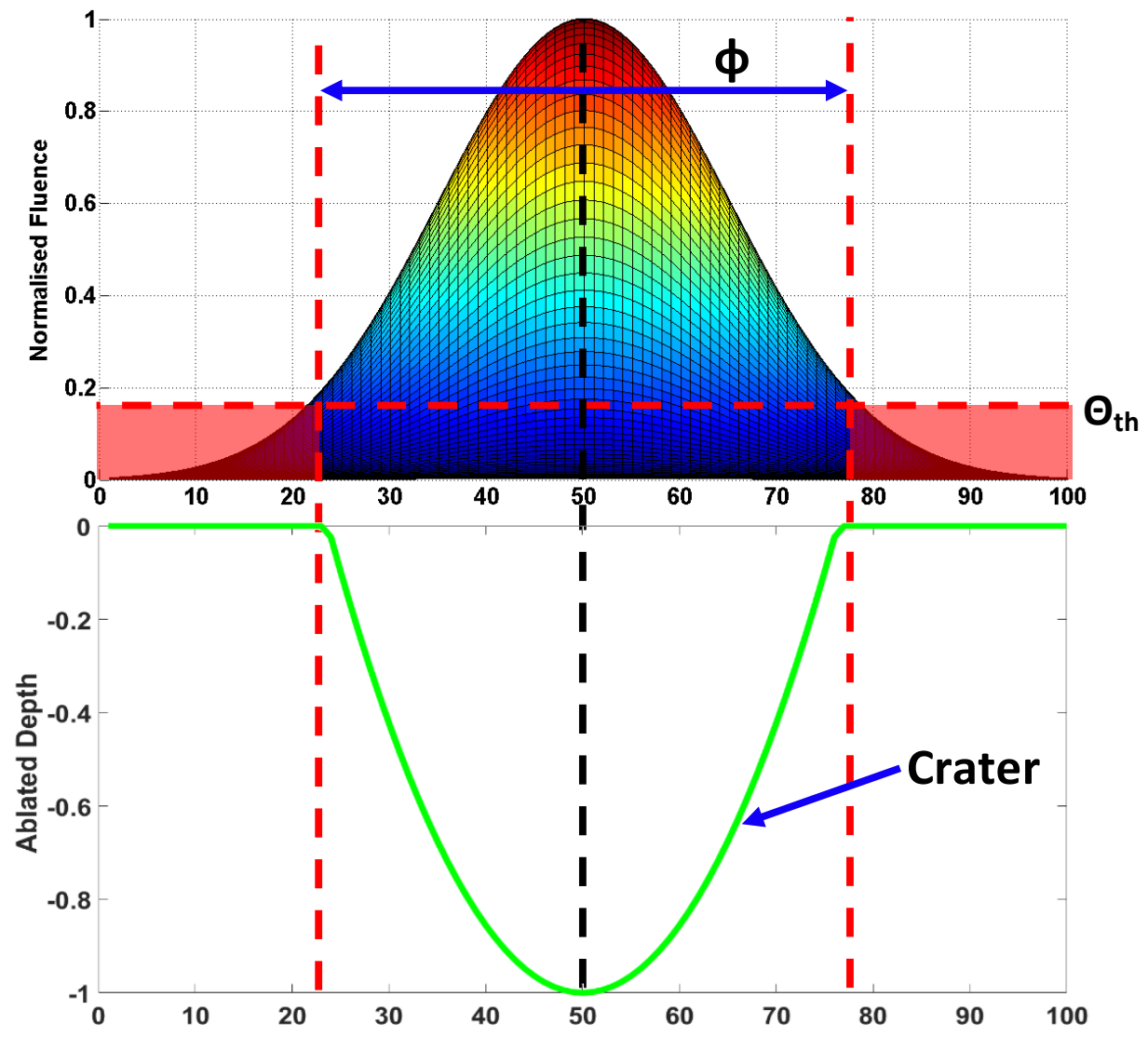

Figure 5 Example of laser beam energy distribution (top) and crater depth profile (bottom), with the red shaded area indicating fluence below the ablation threshold level 


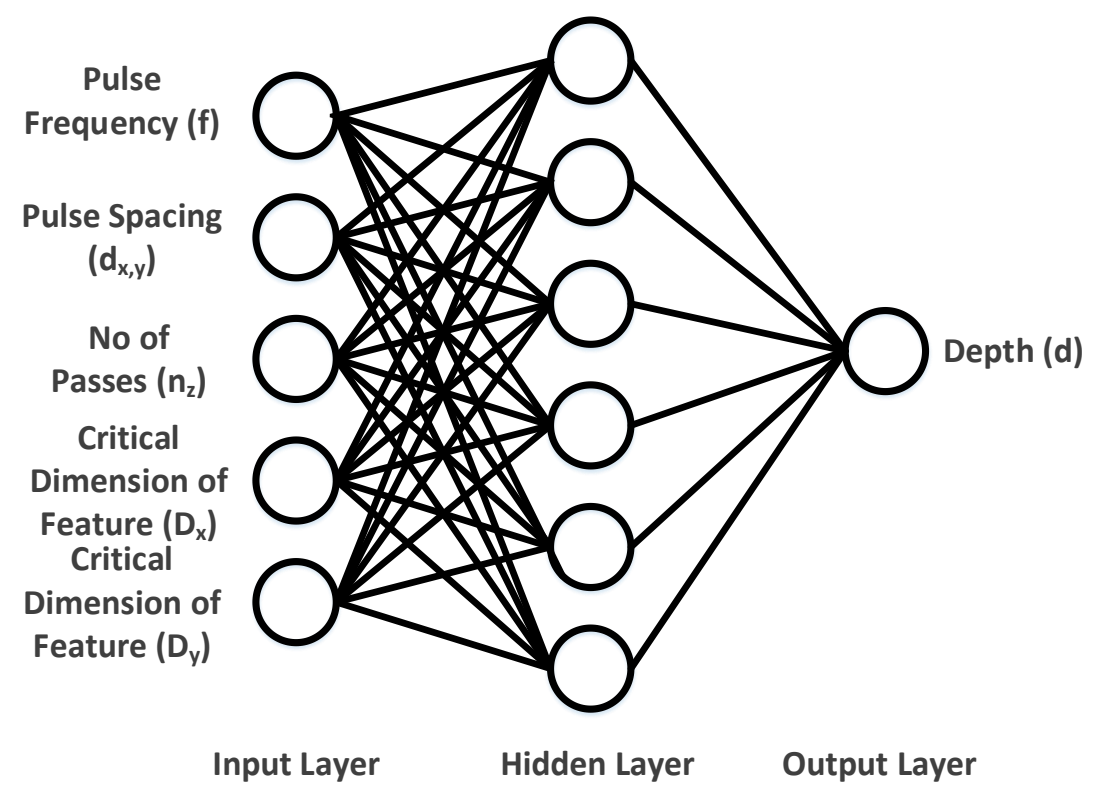

Figure 6 Schematic of the artificial neural network for the prediction of the ablation depth in onthe-fly laser machining 


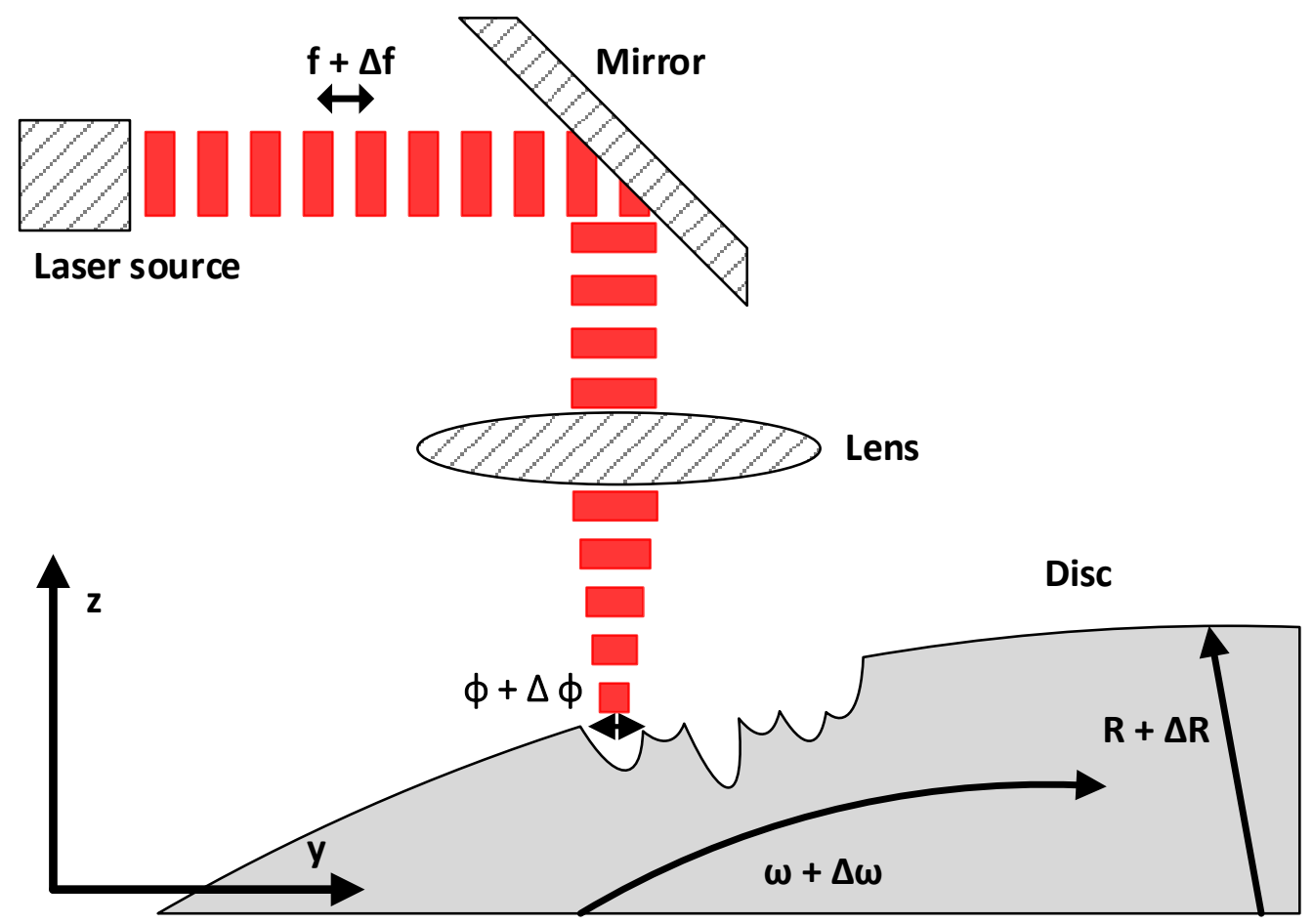

Figure 7 Schematic of the error sources in circumferential (y) direction 
Journal of Manufacturing Science and Engineering

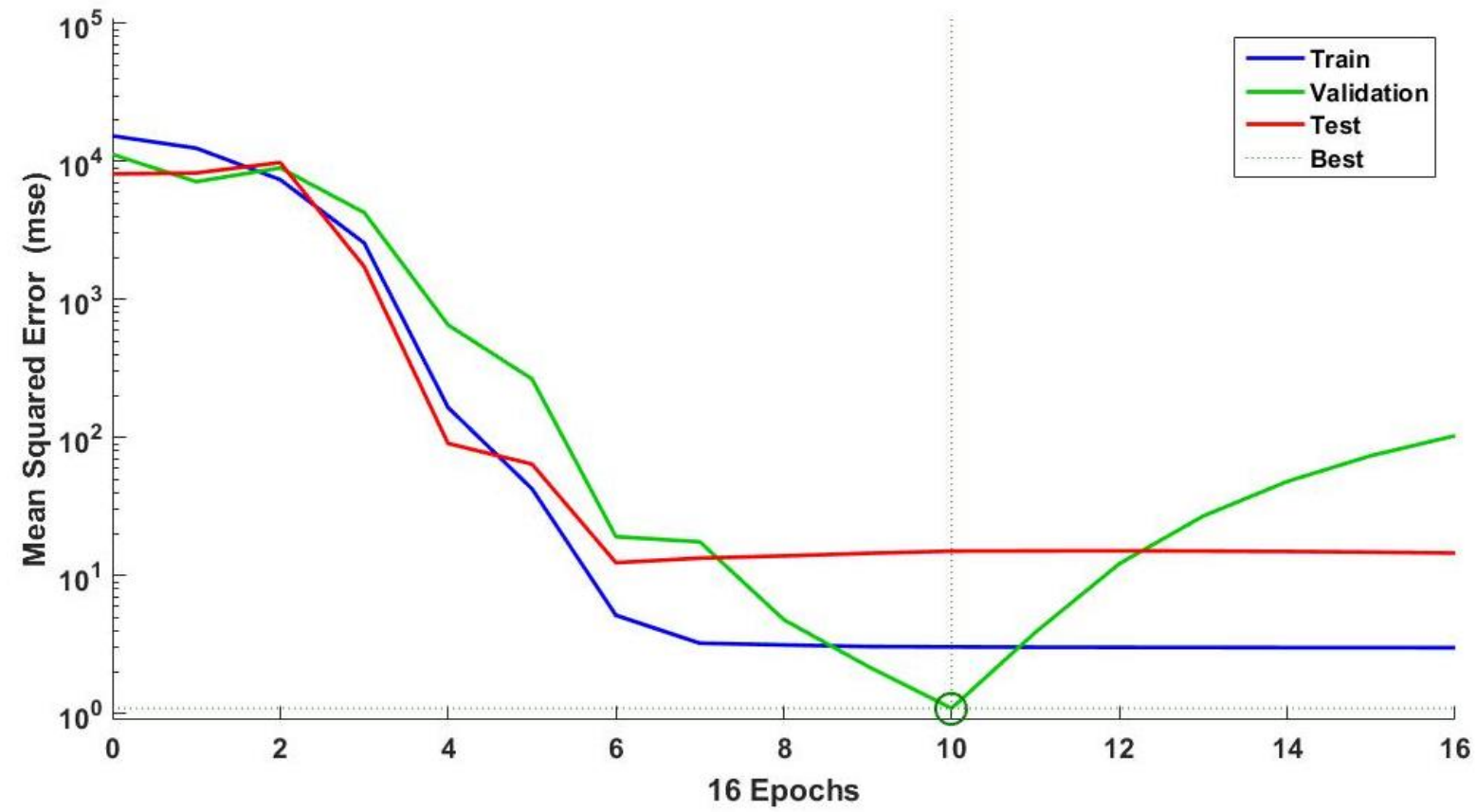

Figure 8 Validation performance of the trained neural network showing the point on which minimum error between the predicted and measured ablated depth was achieved 

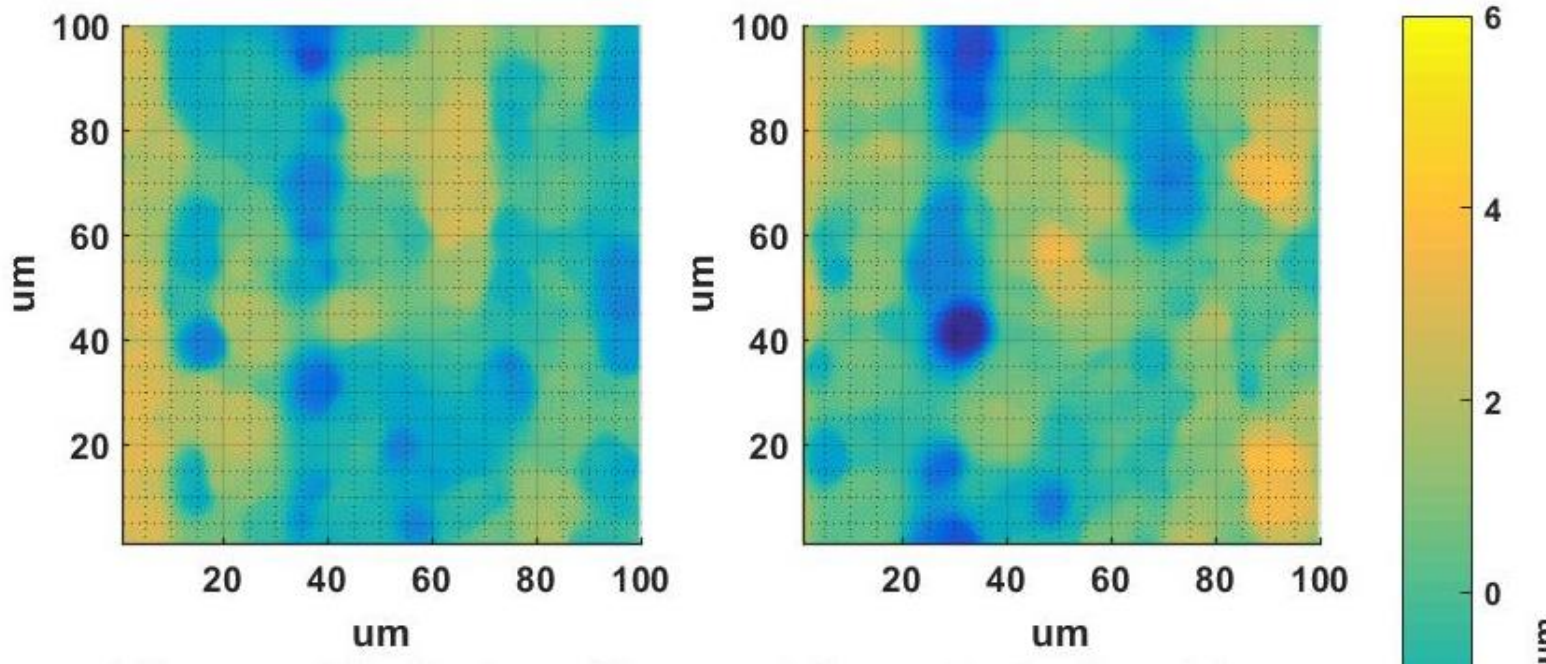

a) Hexagonal Packaging - 1 Layer

c) Square Packaging - 1 Layer
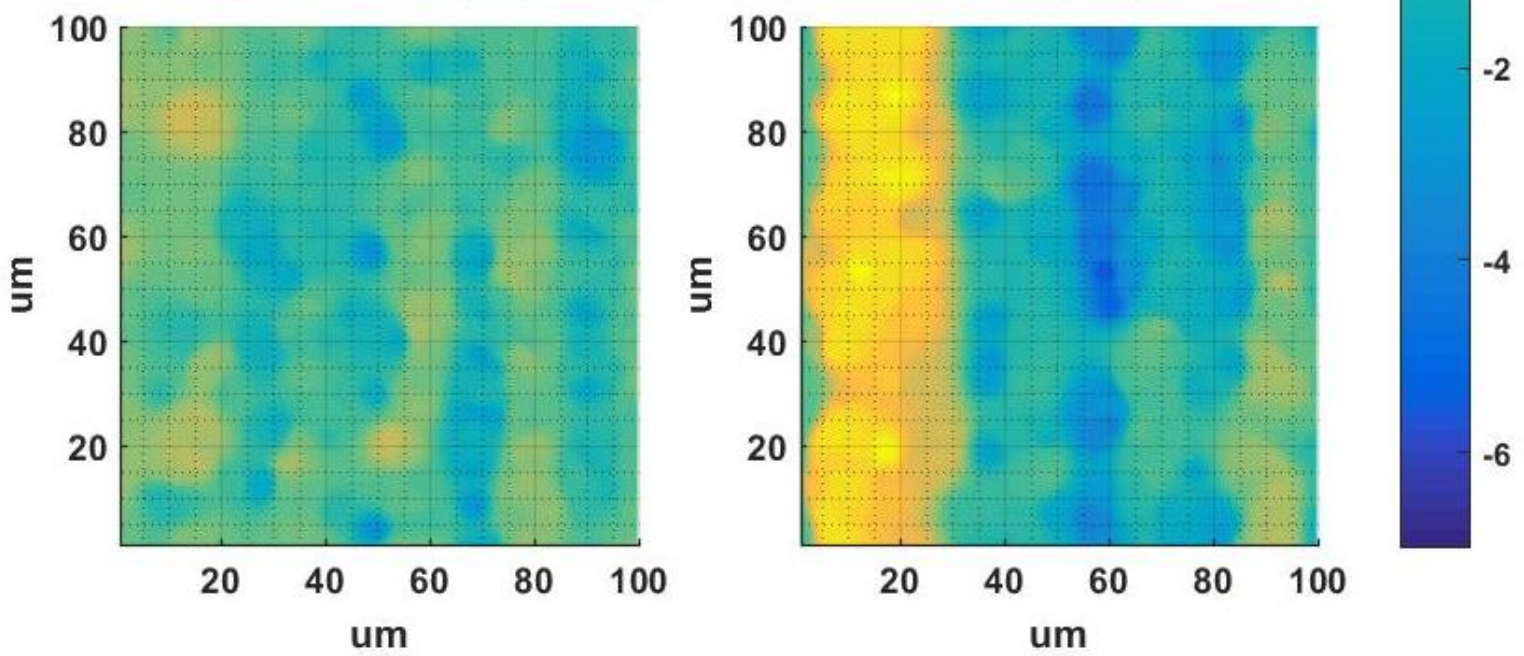

b) Hexagonal Packaging - $\mathbf{3}$ Layers

d) Square Packaging - 3 Layers

Figure 9 Comparison between the surface finishes of a) 1 layer hexagonal pulse placement, b) 3 layers hexagonal pulse placement, c) 1 layer square pulse placement, d) 3 layers square pulse placement 


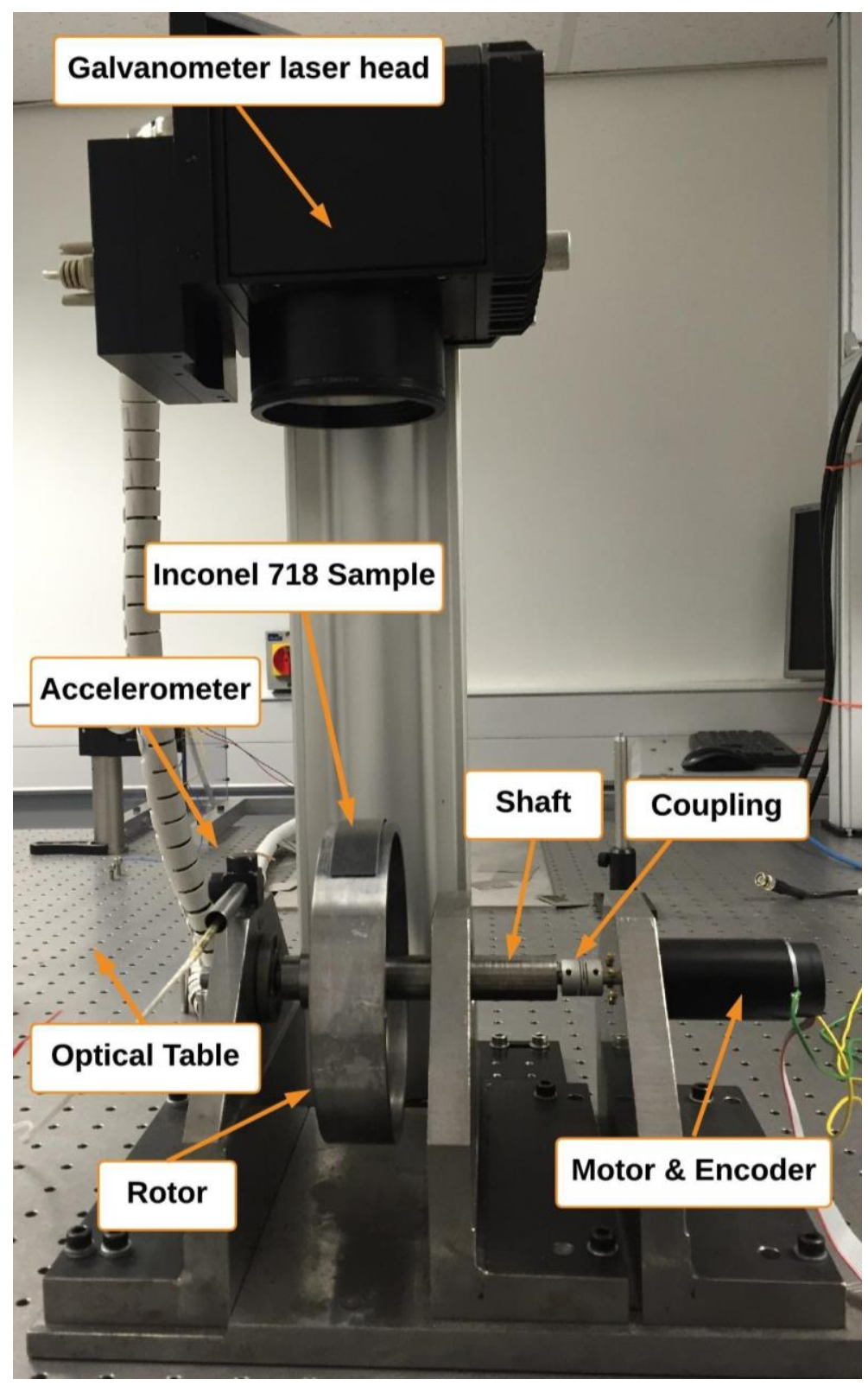

Figure 10 View of the testing rig setup 
Journal of Manufacturing Science and Engineering

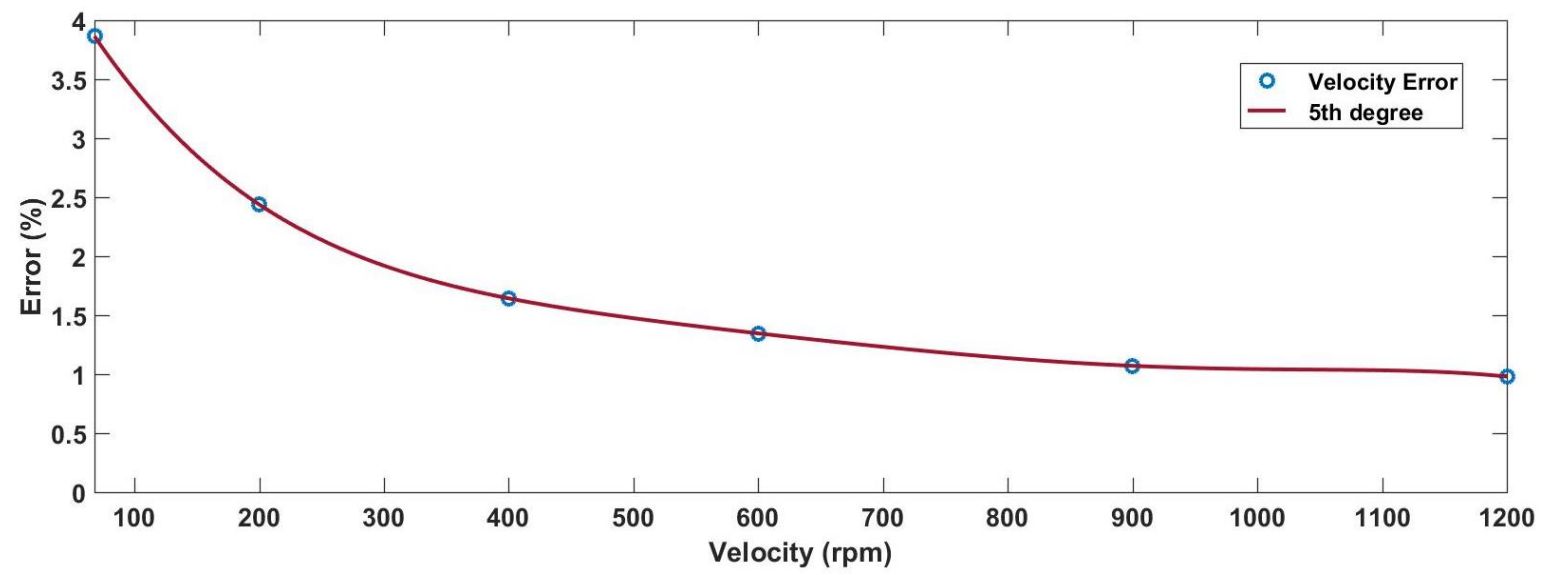

Figure 11 Velocity error dependent on rotor speed 


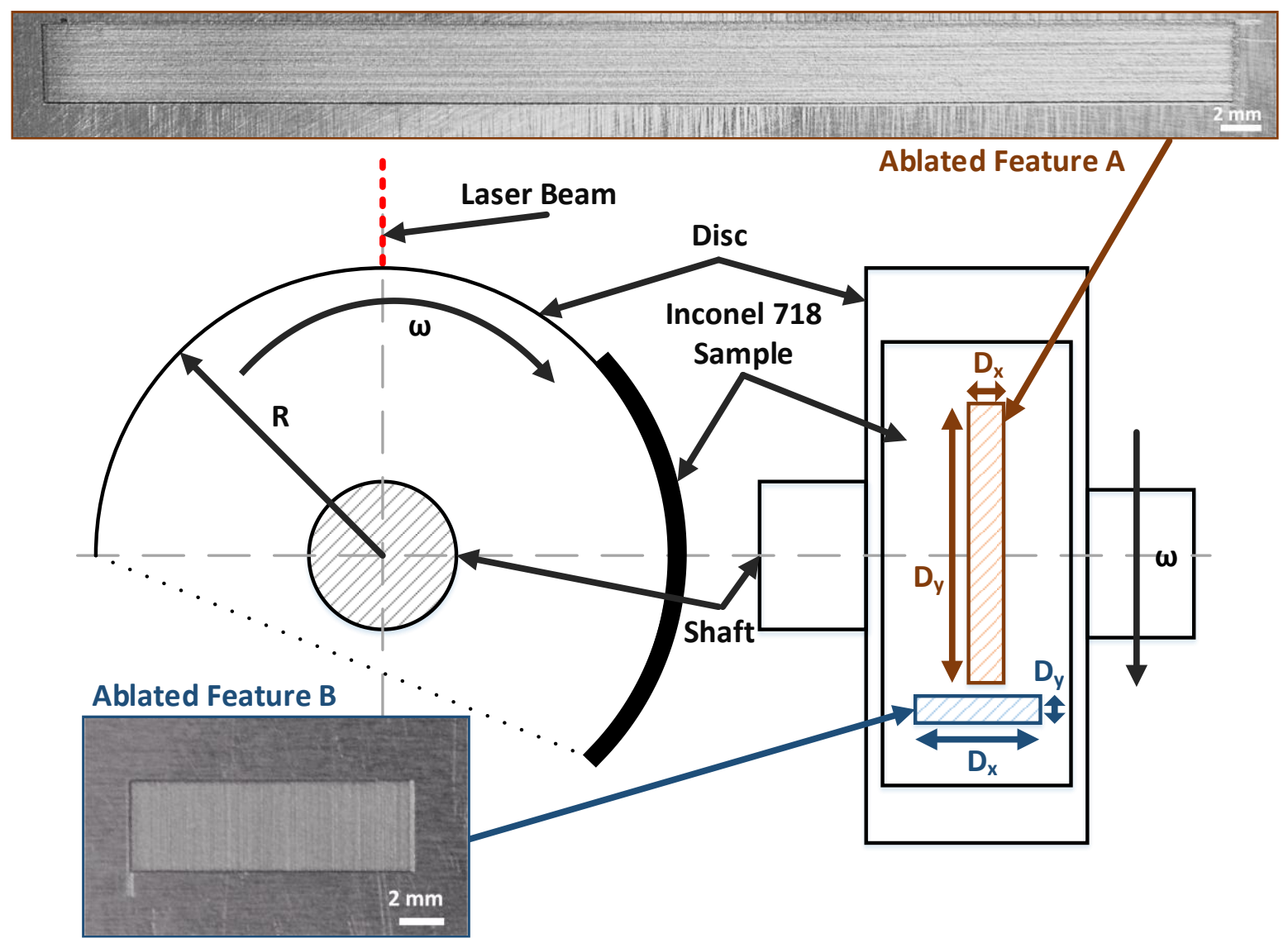

Figure 12 Rotary stage with Inconel 718 sample attached (see experimental set-up in Figure 10) 
Journal of Manufacturing Science and Engineering

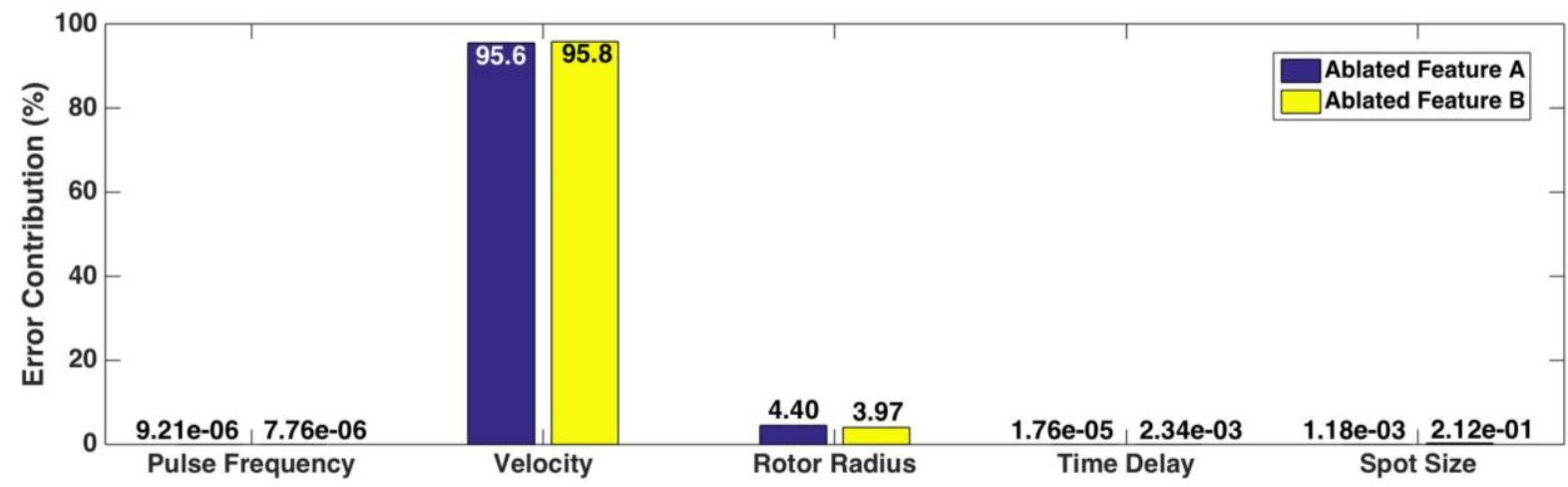

Figure 13 Error contribution of ablated feature $A$ and $B$ to $\Delta D_{y}$ (see Eq. (18)) 
Journal of Manufacturing Science and Engineering

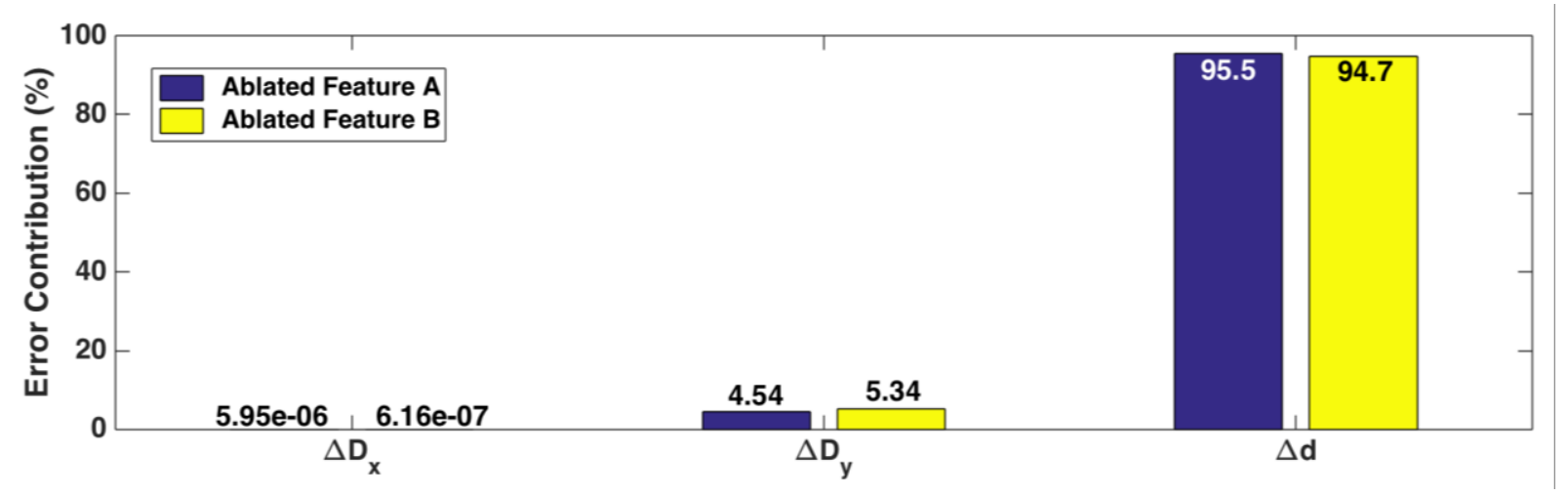

Figure 14 The error contribution of ablated feature A and B to $\Delta m$ (see Eq. (20)) 
Journal of Manufacturing Science and Engineering

Table 1 Variation intervals for the neural network input parameters

\begin{tabular}{|l|l|l|}
\hline Input Parameter & Minimum & Maximum \\
\hline Frequency $(f)$ & $28 \mathrm{kHz}$ & $50 \mathrm{kHz}$ \\
\hline Pulse Spacing $\left(d_{x, y}\right)$ & $10 \mu \mathrm{m}$ & $40 \mu \mathrm{m}$ \\
\hline No of Layers $\left(n_{z}\right)$ & 1 & 222 \\
\hline Critical Dimension $D_{y}$ & $2 \mathrm{~mm}$ & $60 \mathrm{~mm}$ \\
\hline Critical Dimension $D_{x}$ & $2 \mathrm{~mm}$ & $60 \mathrm{~mm}$ \\
\hline
\end{tabular}


Journal of Manufacturing Science and Engineering

Table 2 Experimental and training results of the neural network for Inconel 718 calibration trials, blue shading indicates square ablated patches (i.e. $4 \times 4 \mathrm{~mm}$ ), while orange shading indicates rectangular (i.e. not square) ablated patches

\section{Parameters}

\begin{tabular}{|c|c|c|c|c|c|c|}
\hline $\mathbf{D}_{\mathbf{x}}(\mathrm{mm})$ & $D_{y}(\mathbf{m m})$ & $\mathbf{d}_{x, y}(\mu \mathrm{m})$ & $\mathbf{f}(\mathbf{k H z})$ & $\mathbf{n}_{\mathbf{z}}$ & $\mathbf{d}_{\mathrm{m}}(\boldsymbol{\mu m})$ & $d_{p}(\mu m)$ \\
\hline 4 & 4 & 40 & 50 & 1 & -0.3 & -0.43 \\
\hline 4 & 4 & 40 & 50 & 25 & -3.08 & -3.07 \\
\hline 4 & 4 & 40 & 50 & 3 & -0.11 & -0.67 \\
\hline 4 & 4 & 40 & 50 & 9 & -1.69 & -1.35 \\
\hline 4 & 4 & 40 & 45 & 1 & -0.12 & -0.14 \\
\hline 4 & 4 & 40 & 45 & 25 & -2.78 & -2.73 \\
\hline 4 & 4 & 40 & 45 & 3 & -0.62 & -0.37 \\
\hline 4 & 4 & 40 & 45 & 9 & -1.14 & -1.04 \\
\hline 4 & 4 & 40 & 40 & 1 & 0.05 & -0.05 \\
\hline 4 & 4 & 40 & 40 & 25 & -2.97 & -2.59 \\
\hline 4 & 4 & 40 & 40 & 3 & -0.19 & -0.27 \\
\hline 4 & 4 & 40 & 40 & 9 & -0.57 & -0.94 \\
\hline 4 & 4 & 40 & 35 & 1 & -0.37 & 0.01 \\
\hline 4 & 4 & 40 & 35 & 25 & -2.35 & -2.45 \\
\hline 4 & 4 & 40 & 35 & 3 & -0.23 & -0.21 \\
\hline 4 & 4 & 40 & 35 & 9 & -0.12 & -0.85 \\
\hline 4 & 4 & 20 & 50 & 1 & -0.44 & 0.11 \\
\hline 4 & 4 & 20 & 50 & 25 & -15.1 & -15.06 \\
\hline 4 & 4 & 20 & 50 & 3 & -1.76 & -1.45 \\
\hline 4 & 4 & 20 & 50 & 9 & -5.69 & -5.70 \\
\hline 4 & 4 & 20 & 45 & 1 & -0.2 & -0.02 \\
\hline 4 & 4 & 20 & 45 & 25 & -14.67 & -14.41 \\
\hline 4 & 4 & 20 & 45 & 3 & -1.55 & -1.50 \\
\hline 4 & 4 & 20 & 45 & 9 & -4.99 & -5.52 \\
\hline 4 & 4 & 20 & 40 & 1 & -0.28 & -0.11 \\
\hline 4 & 4 & 20 & 40 & 25 & -13.45 & -13.64 \\
\hline 4 & 4 & 20 & 40 & 3 & -1.33 & -1.50 \\
\hline 4 & 4 & 20 & 40 & 9 & -4.71 & -5.29 \\
\hline 4 & 4 & 20 & 35 & 1 & -0.78 & 0.08 \\
\hline 4 & 4 & 20 & 35 & 25 & -12.47 & -12.22 \\
\hline 4 & 4 & 20 & 35 & 3 & -1.33 & -1.21 \\
\hline 4 & 4 & 20 & 35 & 9 & -4.6 & -4.70 \\
\hline 4 & 4 & 10 & 50 & 1 & -2.26 & -0.45 \\
\hline 4 & 4 & 10 & 50 & 25 & -62.89 & -63.72 \\
\hline 4 & 4 & 10 & 50 & 3 & -8.14 & -6.84 \\
\hline 4 & 4 & 10 & 50 & 9 & -25.24 & -24.55 \\
\hline 4 & 4 & 10 & 45 & 1 & -1.87 & -1.15 \\
\hline
\end{tabular}


Journal of Manufacturing Science and Engineering

\begin{tabular}{|c|c|c|c|c|c|c|}
\hline 4 & 4 & 10 & 45 & 25 & -63.48 & -61.36 \\
\hline 4 & 4 & 10 & 45 & 3 & -7.59 & -7.25 \\
\hline 4 & 4 & 10 & 45 & 9 & -23.37 & -24.10 \\
\hline 4 & 4 & 10 & 40 & 1 & -1.6 & -1.62 \\
\hline 4 & 4 & 10 & 40 & 25 & -58.05 & -58.55 \\
\hline 4 & 4 & 10 & 40 & 3 & -6.95 & -7.40 \\
\hline 4 & 4 & 10 & 40 & 9 & -24.76 & -23.37 \\
\hline 4 & 4 & 10 & 35 & 1 & -1.62 & -1.23 \\
\hline 4 & 4 & 10 & 35 & 25 & -53.41 & -53.75 \\
\hline 4 & 4 & 10 & 35 & 3 & -6.69 & -6.65 \\
\hline 4 & 4 & 10 & 35 & 9 & -22.84 & -21.54 \\
\hline 4 & 4 & 11 & 48 & 134 & -274.62 & -274.51 \\
\hline 4 & 12 & 12 & 44 & 68 & -111.88 & -100.43 \\
\hline 4 & 24 & 15 & 44 & 154 & -152.57 & -152.52 \\
\hline 2 & 30 & 17 & 45 & 222 & -144.43 & -146.46 \\
\hline 2 & 30 & 17 & 45 & 222 & -146.47 & -146.46 \\
\hline 4 & 27 & 13 & 49 & 96 & -125.45 & -118.14 \\
\hline 4 & 60 & 15 & 49 & 167 & -157.65 & -158.48 \\
\hline 4 & 60 & 15 & 48 & 56 & -42.72 & -42.64 \\
\hline 4 & 27 & 13 & 49 & 96 & -110.75 & -118.14 \\
\hline
\end{tabular}


Journal of Manufacturing Science and Engineering

Table 3 Root mean square (Sq) area roughness (ISO 25178) comparison between hexagonal and square pulse placement

\begin{tabular}{|l|l|l|l|}
\hline & Square placement & Hexagonal & Reduction $(\%)$ \\
\hline 1 Layer $-\mathbf{S q}(\boldsymbol{\mu m})$ & 2.18 & 1.81 & -16.97 \\
\hline 3 Layers $-\mathbf{S q}(\boldsymbol{\mu m})$ & 1.17 & 1.02 & -12.82 \\
\hline
\end{tabular}


Journal of Manufacturing Science and Engineering

Table 4 Parameters for the on-the-fly laser machining and error prediction trials

\begin{tabular}{|c|c|c|c|c|c|}
\hline Ablated features & $\mathbf{D}_{\mathbf{x}}(\mathbf{m m})$ & $D_{y}(\mathbf{m m})$ & m (mg) & $m_{d}(\mathrm{mg})$ & $\Delta m_{d}(m g)$ \\
\hline A - Long feature & 4 & 60 & 7538 & 84 & 1 \\
\hline B - Wide feature & 12 & 4 & 7539 & 16 & 1 \\
\hline
\end{tabular}


Journal of Manufacturing Science and Engineering

Table 5 Errors associated with the pulse generation and placement

\begin{tabular}{|l|l|l|l|l|}
\hline Variable & $\begin{array}{l}\text { Standard } \\
\text { deviation } \\
\text { error }\end{array}$ & Description & $\begin{array}{l}\text { Error } \\
\text { distribution }\end{array}$ & $\begin{array}{l}\text { Method of } \\
\text { measuring }\end{array}$ \\
\hline$\Delta \mathrm{t}_{\mathrm{d}}$ & $\pm 0.012 \mathrm{~ms}$ & $\begin{array}{l}\text { Time delay due to } \\
\text { triggering }\end{array}$ & Gaussian & $\begin{array}{l}\text { Empirical: } \\
\text { circumferential } \\
\text { distance } \\
\text { travelled }\end{array}$ \\
\hline$\Delta \omega$ & $\begin{array}{l}\text { Velocity } \\
\text { dependent } \\
\text { (see Eq. }(24), \\
\text { Figure } 11)\end{array}$ & Angular velocity & Normal & $\begin{array}{l}\text { Empirical: } \\
\text { encoder output }\end{array}$ \\
\hline$\Delta \mathrm{R}$ & $\begin{array}{l} \pm 0.05 \mathrm{~mm} \\
\text { Rotor radius }\end{array}$ & Normal & $\begin{array}{l}\text { Empirical: } \\
\text { digital caliper } \\
\text { ruler }\end{array}$ \\
\hline$\Delta \mathrm{f}$ & $\pm 0.5 \mathrm{~Hz}$ & Frequency & Normal & Datasheet \\
\hline$\Delta \phi$ & $\pm 5 \%$ & Pulse diameter & Gaussian & $\begin{array}{l}\text { Empirical: } \\
\text { CMOS beam } \\
\text { profiler }\end{array}$ \\
\hline$\Delta \mathrm{d}$ & $\pm 12.66 \%$ & Feature depth & Normal & $\begin{array}{l}\text { Empirical: } \\
\text { calibration } \\
\text { trials }\end{array}$ \\
\hline$\Delta \mathrm{D}_{\mathrm{x}}$ & $\pm 1 \mu \mathrm{m}$ & $\begin{array}{l}\text { Positional } \\
\text { accuracy of } \mathrm{x} \\
\text { mirror movement }\end{array}$ & Normal & Datasheet \\
\hline
\end{tabular}


Journal of Manufacturing Science and Engineering

Table 6 On-the-fly laser machining process parameter optimisation results for ablated feature A and $B$

\begin{tabular}{|l|l|l|l|l|l|l|l|l|l|}
\hline $\begin{array}{l}\text { Ablated } \\
\text { feature }\end{array}$ & $\begin{array}{l}\mathbf{d}_{\mathbf{x}, \mathbf{y}} \\
(\boldsymbol{\mu \mathbf { m }})\end{array}$ & $\mathbf{f}(\mathbf{k H z})$ & $\mathbf{P}(\mathbf{W})$ & $\mathbf{n}_{\mathbf{x}}$ & $\mathbf{n}_{\mathbf{y}}$ & $\mathbf{n}_{\mathbf{z}}$ & $\boldsymbol{\omega}(\mathbf{r p m})$ & $\mathbf{t}(\mathbf{m i n})$ & $\mathbf{m}_{\mathbf{p}}(\mathbf{m g})$ \\
\hline A & 15 & 48 & 18 & 262 & 3996 & 56 & 100 & 146.72 & 83.84 \\
\hline B & 14 & 45 & 17 & 852 & 281 & 30 & 86 & 297.21 & 16.25 \\
\hline
\end{tabular}


Journal of Manufacturing Science and Engineering

Table 7 On-the-fly laser machining results for ablated feature A and B

\begin{tabular}{|l|l|l|l|}
\hline Ablated feature & $\mathbf{m}_{\mathbf{p}} \mathbf{( m g )}$ & $\mathbf{m} \mathbf{( m g )}$ & Error $\mathbf{( \% )}$ \\
\hline A & 83.84 & $85 \pm 2$ & 1.38 \\
\hline B & 16.25 & $13 \pm 2$ & 20.00 \\
\hline
\end{tabular}


Journal of Manufacturing Science and Engineering

Table 8 Error model evaluation results for $\Delta D_{y}$ and $\Delta m$

\begin{tabular}{|l|l|l|l|l|}
\hline \multirow{2}{*}{$\begin{array}{l}\text { Ablated } \\
\text { feature }\end{array}$} & \multicolumn{2}{|c|}{$\Delta \mathbf{D}_{\mathbf{y}}$} & \multicolumn{2}{c|}{$\Delta \mathbf{m}$} \\
\cline { 2 - 5 } & $\mathbf{D}_{\mathbf{y}}(\mathbf{m m})$ & $\mathbf{D}_{\mathbf{y p}}(\mathbf{m m})$ & $\mathbf{m} \mathbf{( m g )}$ & $\mathbf{m}_{\mathbf{p}}(\mathbf{m g})$ \\
\hline $\mathrm{A}$ & $60_{-1.711}^{-1.765}$ & $60 \pm 2.095$ & $83.84_{-0.840}^{+3.160}$ & $83.84 \pm 11.01$ \\
\hline $\mathrm{B}$ & $4_{+1.138}^{+1.140}$ & $4 \pm 0.157$ & $16.25_{-5.250}^{-1.250}$ & $16.25 \pm 2.15$ \\
\hline
\end{tabular}

